\title{
Supra-coronary Aortic Banding Improves Right Ventricular Function in Experimental Pulmonary Arterial Hypertension in Rats by Increasing Systolic Right Coronary Artery Perfusion
}

Tian and Xiong: Aortic banding improves RV function in PAH

Lian Tian $^{1 *}, \mathrm{PhD}$, Ping Yu Xiong ${ }^{1,2^{*}}, \mathrm{PhD}, \mathrm{MD}$, Elahe Alizadeh ${ }^{3}, \mathrm{PhD}$, Patricia D.A. Lima ${ }^{3}$,

PhD, François Potus ${ }^{1}$, PhD, Jeffrey Mewburn ${ }^{1}$, Ashley Martin ${ }^{1}$, Kuang-Hueih Chen ${ }^{1}, \mathrm{PhD}$, Stephen L. Archer ${ }^{1,3 \#}$, MD

${ }^{1}$ Department of Medicine, Queen's University, Kingston, ON, Canada

${ }^{2}$ Department of Biomedical and Molecular Sciences, Queen's University, Kingston, ON, Canada

${ }^{3}$ Queen's CardioPulmonary Unit (QCPU), Kingston, ON, Canada

${ }^{*}$ These two authors contributed equally to this project

\# Corresponding author

Stephen L. Archer MD. FRCP(C), FRSC, FAHA, FACC

Tier 1 CRC Mitochondrial Dynamics

Elizabeth Smith Distinguished Professor

Head Department of Medicine, Queen's University

Director Queen's CardioPulmonary Unit

Program Medical Director for Medicine and Cardiac Sciences at KGH, HDH, SMOL

Etherington Hall, Room 3041

94 Stuart St., Kingston, Ontario, Canada, K7L 3N6

Tel: 613-533-2967

Fax: 613-533-6695

Preferred E-mail: stephen.archer@queensu.ca

Abstract word count: 262

Total word count: 5584 


\begin{abstract}
Aim: Pulmonary arterial hypertension (PAH) results in right ventricular (RV) dysfunction due, in part, to RV ischemia. The relative contribution of RV microvascular rarefaction versus reduced right coronary artery perfusion pressure (RCA-PP) to RV ischemia is unknown. We hypothesize that increasing RCA-PP improves RV function in PAH by increasing RV systolic perfusion.
\end{abstract}

Methods: Supra-coronary aortic banding (SAB) or sham surgery was performed on male SpragueDawley rats. 7-10 days later, rats received either monocrotaline (MCT; 60mg/kg) or saline. After one month, echocardiography, cardiac catheterization, ${ }^{99 \mathrm{~m}} \mathrm{Tc}$-sestamibi single-photon emission computed tomography (SPECT) and microsphere infusion studies were performed. The RV was harvested for measurement of hypertrophy $(\mathrm{RVH})$, fibrosis and immunoblotting, and the lung was harvested for pulmonary artery (PA) histology.

Results: SAB increased systolic pressures in proximal aorta and systolic RCA-PP in SAB+MCT vs. MCT rats $(114 \pm 12$ vs. $5 \pm 9 \mathrm{mmHg})$, without altering diastolic RCA-PP. SAB+MCT rats had improved RV function versus MCT rats, evident from their significantly increased cardiac output (CO), RV free wall (RVFW) thickening, tricuspid annular plane systolic excursion (TAPSE), and RV-PA coupling indices. RV-PA coupling indices and CO correlated directly with systolic RCAPP. RV perfusion was increased in SAB+MCT vs. MCT rats and correlated well with $\mathrm{CO}$; whereas microvascular rarefaction was unaltered. $\mathrm{SAB}+\mathrm{MCT}$ rats had less $\mathrm{RVH}$ and fibrosis and lower PA pressures versus MCT rats. SAB+MCT rats had significantly lower RV pyruvate kinase muscle isoform 2/1 ratios than MCT rats, consistent with restoration of oxidative metabolism. 
Conclusion: A SAB-induced increase in systolic RCA-PP improves RV perfusion and function in MCT rats. Maintaining systolic RCA perfusion can preserve RV function in PAH.

Keywords: coronary artery, microvascular rarefaction, mitochondrial metabolism, monocrotaline, pyruvate dehydrogenase kinase, single-photon emission computed tomography (SPECT) 


\section{Introduction}

Pulmonary arterial hypertension (PAH) is a subcategory of pulmonary hypertension (WHO

Group I) in which the primary pathology is obstruction and constriction of the pulmonary vasculature, reviewed in $^{1}$. Pulmonary vascular obstruction and constriction increase right ventricular (RV) afterload, and eventually lead to RV dysfunction and failure. The prognosis in PAH is determined by the response of the RV to increased afterload ${ }^{2-9}$. Recently, mitochondrialmetabolic abnormalities, including increased glucose uptake that accompanies a shift to metabolic reliance on uncoupled glycolysis, have been identified in the RV in both PAH patients and animal models of $\mathrm{PAH}^{10-14}$. Impaired mitochondrial metabolism contributes to reduced RV myocyte contractility and $\mathrm{RV}$ dysfunction ${ }^{15}$. A recent transcriptomic analysis of the $\mathrm{RV}$ in the monocrotaline (MCT) model shows the leading pathways that are dysregulated include mitochondrial metabolic, inflammatory, fibrotic and angiogenic pathways ${ }^{13}$. The metabolic remodeling in RV hypertrophy $(\mathrm{RVH})$ associated with $\mathrm{PAH}$ is thought to be initiated by ischemia, which is caused by the combination of reduced right coronary artery (RCA) perfusion pressure $(\text { RCA-PP) })^{16}$ and intramyocardial microvascular rarefaction ${ }^{17,18}$. There is substantial evidence of RV ischemia in human PAH patients, including abnormal RCA flow reserve on cardiac magnetic resonance imaging ${ }^{19}$ and the not-infrequent occurrence of elevated plasma troponin levels ${ }^{20}$. Coronary flow reserve in response to adenosine is also reduced in the RV in $\mathrm{PAH}^{19}$. Finally, RV ischemia is also observed in rats with experimental PAH, induced by $\mathrm{MCT}^{21,22}$ or Sugen-hypoxia ${ }^{18}$.

While most studies focus on enhancing RV function in PAH indirectly, by regressing pulmonary vascular disease, some attempts have been made to directly target the RV and improve its blood supply by promoting angiogenesis ${ }^{23,24}$ or by improving the bioenergetic capacity of RV myocytes by altering glucose or fatty acid oxidation allowing the RV "do more with less", 
reviewed $\mathrm{in}^{17}$. A potentially complementary strategy to improve RV function is to increase epicardial RCA-PP ${ }^{17}$. This has been attempted in both animal studies and in patients with PAH. In animal studies of acute RV failure ${ }^{25}$ and compensated RVH, caused by pulmonary artery banding ${ }^{26}$, RV function was improved by increasing aortic pressure (using phenylephrine and carotid occlusion, respectively). However, there is limited assessment of this strategy in PAH models of chronic RV failure or decompensated RVH. In patients with PAH, infusion of phenylephrine or norepinephrine has been used to transiently increase aortic pressure and thus RCA-PP. Although both interventions increased aortic pressure, both failed to improve RV function ${ }^{27,28}$, perhaps due to concomitant induction of pulmonary vasoconstriction and associated increases in pulmonary vascular resistance $(\mathrm{PVR})$ and pulmonary arterial pressure $(\mathrm{PAP})^{27}$. It is unknown whether a selective and sustained increase in RCA-PP, induced by an isolated increase in aortic pressure, would improve RV function in PAH. In this study we sought to restore RCA systolic perfusion, which is impaired in PAH. The RCA is perfused in both systole and diastole in normal subjects, whilst in PAH patients the systolic component of perfusion is selectively diminished, as RV pressures approach systemic levels of pressure ${ }^{16}$. This proof of concept experiment was also intended to establish the relative importance of epicardial perfusion pressure versus microvascular rarefaction in $\mathrm{RV}$ dysfunction.

We hypothesize that increasing RCA-PP improves RV function in PAH by reducing RV ischemia. We achieved a sustained increase in RCA-PP in the MCT rat model of PAH-associated RV failure using supra-coronary aortic banding (SAB). We show that SAB increased left ventricular (LV) systolic pressure, leading to increases in systolic RCA-PP and flow in MCT rats. Improved coronary perfusion reduced $\mathrm{RVH}$ and improved $\mathrm{RV}$ contractile function without altering RV microvascular rarefaction, adverse pulmonary vascular remodeling or altering LV function. 
These findings establish the primary importance of impaired RCA systolic coronary perfusion pressure to the pathogenesis of RV failure in PAH. 


\section{Results}

\section{$\mathrm{SAB}$ reduces $\mathrm{RV}$ hypertrophy in $\mathrm{SAB}+\mathrm{MCT}$ vs. MCT rats}

MCT significantly reduced body weight versus Control (402 \pm 12 vs. $496 \pm 11$ g, respectively) and significantly increased both absolute RV weight $(0.59 \pm 0.02$ vs. $0.28 \pm 0.02 \mathrm{~g}$ for MCT vs. Control) and the RV/body weight ratio $(0.15 \pm 0.008 \%$ vs. $0.055 \pm 0.003 \%$ for MCT vs. Control) (Fig. 2). SAB did not significantly change body weight of either SAB or SAB+MCT rats, but caused a significant increase in $\mathrm{LV}+$ septum $(\mathrm{LV}+\mathrm{S})$ weight. $\mathrm{SAB}+\mathrm{MCT}$ rats had significant reduction in both $\mathrm{RV}$ weight $(0.49 \pm 0.02$ vs. $0.59 \pm 0.02 \mathrm{~g}$ for $\mathrm{SAB}+\mathrm{MCT}$ vs. MCT) and the RV/body weight ratio $(0.12 \pm 0.003 \%$ vs. $0.15 \pm 0.008 \%$ for SAB+MCT vs. MCT) (Fig. 2).

\section{SAB increases RCA-PP and improves RV contractile function and cardiac output in SAB+MCT vs. MCT rats}

At week 4 post MCT injection, pulmonary hypertension with RVH was evident in MCT versus Control rats on echocardiography, demonstrated both by a shortened pulmonary artery (PA) acceleration time (PAAT) and an increased diastolic RV free wall (RVFW) thickness with reduced RVFW thickening and tricuspid annular plane systolic excursion (TAPSE) (Fig. 3A). Compared to Control, the relatively mild SAB alone resulted in a trend toward RVH which was associated with mild pulmonary hypertension and a mild reduction in RV function, evident as shortened PAAT and reduced RVFW thickening and TAPSE (Fig. 3A). In contrast in the SAB+MCT group, RV function was improved and RVH was reduced compared to MCT group, evident as a reduction in diastolic RVFW thickness $(1.18 \pm 0.06$ vs. $1.52 \pm 0.12 \mathrm{~mm})$ and increased RVFW thickening ( $45 \pm 4$ vs. $26 \pm 2 \%)$ and TAPSE (2.32 \pm 0.07 vs. $2.08 \pm 0.05 \mathrm{~mm})$. Moreover, PAAT was prolonged 
in the $\mathrm{MCT}+\mathrm{SAB}$ group $(24 \pm 0.7$ vs. $21 \pm 0.9 \mathrm{~ms}$ for $\mathrm{SAB}+\mathrm{MCT}$ vs. MCT), which was compatible with a mild reduction in pulmonary hypertension (Fig. 3A).

Representative pressure traces in aorta, proximal and distal to the band in a SAB rat, and in the RV (of a MCT rat) are presented in Supplemental Fig. S1. These traces illustrate how the pressure data were extracted. MCT increased RV systolic pressure (RVSP) and slightly decreased LV systolic pressure (LVSP) without altering ascending aortic pressure or LV end-diastolic pressure (LVEDP) versus Control (Supplemental Fig. S2\&S3). SAB increased systolic pressures both in the ascending aorta and LV (both in SAB and SAB+MCT groups) along with a transbanding systolic pressure gradient of $116 \pm 18 \mathrm{mmHg}$ in $\mathrm{SAB}$ rats and $88 \pm 8 \mathrm{mmHg}$ in $\mathrm{SAB}+\mathrm{MCT}$ rats; in contrast, SAB did not alter aortic or LV diastolic pressures (Supplemental Fig. S2). In addition, this mild degree of SAB did not change RVSP or RV end-diastolic pressure (RVEDP) in either SAB or SAB+MCT rats (Supplemental Fig. S3).

Representative pressure traces in the RV and the aorta proximal to the band for each group are presented in Fig. 3B. MCT caused a significant decrease in systolic RCA-PP and a mild decrease in diastolic RCA-PP (Fig. 3C). In 3 out of 7 MCT rats, RV pressure was even higher than aortic pressure during systole. As a result, the calculated systolic RCA-PP was negative for these 3 rats, indicating the lack of driving gradient for systolic RV perfusion. SAB significantly increased systolic RCA-PP in both SAB (167 \pm 20 vs. $84 \pm 1 \mathrm{mmHg}$ for SAB vs. Control) and $\mathrm{SAB}+\mathrm{MCT}$ rats $(114 \pm 12$ vs. $5 \pm 9 \mathrm{mmHg}$ for SAB+MCT vs. MCT $)$ and slightly decreased diastolic RCA-PP in SAB rats (70 \pm 5 vs. $88 \pm 3 \mathrm{mmHg}$ for $\mathrm{SAB}$ vs. Control) without altering diastolic RCA$\mathrm{PP}$ in $\mathrm{SAB}+\mathrm{MCT}$ rats (Fig. 3C).

Compared to the MCT group, rats from SAB+MCT group had significantly higher LV cardiac output $(\mathrm{CO})$ measured from catheterization $(82 \pm 11$ vs. $38 \pm 9 \mathrm{~mL} / \mathrm{min})$ (Fig. 3C). MCT rats 
also had significantly lower heart rate than Control rats ( $265 \pm 10$ vs. $335 \pm 14$ beats/min), but there was no difference in the heart rate between SAB+MCT and MCT groups $(277 \pm 16$ vs. $265 \pm 10$ beats/min) (Supplemental Fig. S3). Pulmonary vascular resistance (PVR) was significantly reduced in the SAB+MCT group compared to the MCT group $[0.29 \pm 0.04$ vs. $1.43 \pm 0.28$ $\mathrm{mmHg} /(\mathrm{mL} / \mathrm{min})]$ (Supplemental Fig. S3).

\section{RV-PA coupling is improved in MCT+SAB vs. MCT rats}

RV-PA coupling assessed either by the ratio of RVFW thickening to RVSP or the ratio of TAPSE to RVSP was significantly reduced in the MCT versus Control group. RV-PA coupling was improved in the SAB+MCT versus MCT group (Fig. 4A). RV end-systolic elastance ( $\mathrm{E}_{\mathrm{es}}$ ), estimated from single-beat method, did not change significantly with SAB or MCT treatment. In contrast, pulmonary effective arterial elastance $\left(E_{a}\right)$, a measure of arterial load, increased significantly in MCT versus Control group, consistent with the observed rise in PVR. SAB tended to reduce the $\mathrm{E}_{\mathrm{a}}(\mathrm{P}=0.056)$ in $\mathrm{SAB}+\mathrm{MCT}$ versus $\mathrm{MCT}$ group. $\mathrm{RV}-\mathrm{PA}$ coupling, $\mathrm{E}_{\mathrm{es}} / \mathrm{E}_{\mathrm{a}}$, which was decreased in the MCT group, was restored in SAB+MCT rats (Fig. 4B). RV Ees $/ \mathrm{E}_{\mathrm{a}}$ correlates well with the two other methods of measuring RV-PA coupling, namely RVFW thickening/RVSP and TAPSE/RVSP (Supplemental Fig. S4). LV E es did not change significantly with SAB or MCT treatment. As expected, systemic $E_{a}$ increased in the SAB group (vs. control group) and in the $\mathrm{SAB}+\mathrm{MCT}$ group (vs. the MCT group) (Fig. 4C). Left ventricular-vascular coupling, $\mathrm{E}_{\mathrm{es}} / \mathrm{E}_{\mathrm{a}}$, decreased in MCT versus Control group but was not significantly altered in SAB+MCT versus MCT group (Fig. 4C). 
Improved RV contractility and $\mathrm{CO}$ in SAB+MCT vs. MCT rats correlate with improved systolic RCA-PP

Linear regression analysis reveals strong positive correlation between systolic (but not diastolic) RCA-PP and RV contractility indices (RVFW thickening, TAPSE and RV end-systolic elastance, $\mathrm{E}_{\mathrm{es}}$ ) (Fig. 5). This indicates that SAB-induced augmentation of systolic RCA-PP is associated with better RV contractility. Likewise, LV CO (measured from catheterization) also correlates better with systolic RCA-PP than with diastolic RCA-PP (Fig. 5).

\section{$\mathrm{RV}$ perfusion is increased in MCT+SAB vs. MCT rats}

Quantitative single-photon emission computed tomography (SPECT) imaging showed that MCT rats had marked reduction in ${ }^{99 m}$ Tc-sestamibi $\left({ }^{99 m} \mathrm{Tc}-\mathrm{MIBI}\right)$ uptake in both $\mathrm{RV}$ and $\mathrm{LV}$ during both systole and diastole compared to control rats. Myocardial perfusion was increased in SAB+MCT group in both RV and LV during both systole and diastole versus MCT group (Fig. 6A, Supplemental Videos S1-4). RV radioactivity uptake during both systole and diastole are well correlated with RV CO estimated from echocardiography (Fig. 6B).

In addition, study on infusion of microspheres into hearts from a limited number of rats shows that the number of microspheres in RV microvasculature was reduced in MCT versus Control group, and increased in SAB+MCT versus MCT group (Fig. 6C\&D). Note that one control rat displayed an arrhythmia immediately after the injection of microspheres and this invalidated use of data from this rat.

\section{RV fibrosis and RV myocyte size are reduced in SAB+MCT vs. MCT rats}


$\mathrm{RV}$ histology revealed marked reduction of fibrotic area in SAB+MCT versus MCT group $(12 \pm 6$ vs. $23 \pm 3 \%)$ (Fig. $7 \mathrm{~A})$. Rats in the SAB+MCT group had a similar extent of fibrosis as rats in the SAB group, and this was not increased compared to the Control group (Fig. 7A). In addition, the cross-sectional area of individual RV myocyte was significantly greater in MCT versus Control group $\left(1040 \pm 39\right.$ vs. $\left.564 \pm 26 \mu \mathrm{m}^{2}\right)$ and reduced in the $\mathrm{SAB}+\mathrm{MCT}\left(564 \pm 21 \mu \mathrm{m}^{2}\right)$ versus MCT group (Fig. 7B). The individual RV myocyte size in $\mathrm{SAB}+\mathrm{MCT}$ group was comparable to the Control and SAB groups (Fig. 7B).

\section{SAB+MCT rats have similar RV microvasculature and adverse pulmonary vascular remodeling as MCT rats}

Immunofluorescence staining on RV with CD31 shows that the capillary density was significantly reduced in MCT versus Control group but was not changed by SAB, in either SAB or $\mathrm{SAB}+\mathrm{MCT}$ rats (Fig. 7C). Likewise, quantitative lung histology revealed that rats in the MCT group had significantly higher number of small muscularized pulmonary vessels $(9.4 \pm 1.0$ vs. $3.5 \pm 0.6$ per $\left.4.9 \mathrm{~mm}^{2}\right)$ and higher medial wall thickness to external diameter ratio $(25 \pm 1 \%$ vs. $9 \pm 1 \%)$ compared to Control group (Fig. 7D), and both were not different in the MCT+SAB group (Fig. 7D).

\section{PKM2/PKM1 ratio is reduced in MCT+SAB vs. MCT RV}

Immunoblotting analysis shows that MCT caused an increase in pyruvate kinase muscle isoform 2 (PKM2) and a reduction in PKM1 in RV, resulting in an increase in the PKM2/PKM1 ratio, versus Control group (Fig. 8). In PBS-injected rats SAB did not alter the PKM2 or PKM1 level or the PKM2/PKM1 ratio; while in MCT-injected rats SAB did not change PKM2 level, but 
restored PKM1 level and the PKM2/PKM1 ratio (Fig. 8). In addition, the ratio of phosphorylated pyruvate dehydrogenase (P-PDH) to total PDH tended to decrease in SAB+MCT versus MCT RV $(\mathrm{P}=0.24$; Supplemental Fig. S5). 


\section{Discussion}

This study addresses the longstanding question whether RV dysfunction in PAH relates to RV ischemia and if so whether this primarily reflects impairment of epicardial RCA blood flow or RV microvascular rarefaction. In a well-accepted preclinical model of PAH in which RV impairment is prominent (the MCT model), we show that RCA-PP is impaired in systole and demonstrate that this impairs RV function. We further show that restoring the systolic RCA-PP, using a mild SAB (which does cause mild Group 2 PH), improves RCA blood flow and RV function without altering RV microvascular rarefaction. Thus while RV microvascular rarefaction occurs in preclinical PAH, as we confirm in the current study, the impairment of RCA systolic flow significantly contributes to RV dysfunction, independent of changes in microvascular rarefaction.

In the normal heart, LV myocardial perfusion primarily occurs in diastole because the aortic root-LV pressure gradient is small in systole. In contrast, due to low RV pressures relative to aortic root pressures, RCA perfusion occurs both in diastolic and systolic phases ${ }^{29}$. In PAH patients with elevated RV pressure, decreased systolic RCA-PP and RCA blood flow have been observed $^{16}$. It is known that reducing RCA perfusion pressure below $50 \mathrm{mmHg}$ in conscious canines acutely impairs RV function ${ }^{30}$. As in patients with PAH, MCT rats with PAH had reduced RV perfusion pressures (RCA-PP) both in diastole and systole. This was closely correlated with, and resulted primarily from, increases in RV diastolic and systolic pressures, exacerbated slightly by small decreases in aortic diastolic and systolic pressures (Fig. 3, Supplemental Figs. S2\&S3). Since the moderate $\mathrm{SAB}$ we induced caused a significant increase in aortic systolic pressure proximal to the band, it is not surprising that $\mathrm{SAB}+\mathrm{MCT}$ rats had, relative to MCT rats, a greatly 
increased systolic RCA-PP (Fig. 3, Supplemental Figs. S2\&S3). We also noted a slight but potentially beneficial decrease in RVSP in MCT+SAB rats (Supplemental Fig. S3).

The consequence of the increased RCA-PP in systole is that RV perfusion was improved both in systole and diastole, as confirmed by ${ }^{99 m}$ Tc-MIBI SPECT image technique (Fig. 6A) and microsphere perfusion study in RV (Fig. 6C\&D). The observed improvement of RV perfusion in diastole (Fig. 6A) is probably due to the fact that SAB induced slight increase in diastolic RCAPP (Fig. 3C) and the enhanced systolic RCA blood flow carried over into the diastolic phase. As a result of the increase in RV perfusion by $\mathrm{SAB}$, several benefits are observed.

First, compared to sham-treated MCT rats, there was less RVH in SAB-treated MCT rats (Figs. 2, 3 \&7B). A similar reduction in RVH has been reported in rabbits with isolated RV hypertension induced by pulmonary artery banding $(\mathrm{PAB})$ that were treated with $\mathrm{SAB}^{26}$. The decrease in RVH in SAB-treated MCT rats may be due to the improved RV function (discussed below) which leads to regression in the compensatory hypertrophic program that originally compensates for high RV afterload. Indeed, RV mass is inversely correlated to RV function in PAH patients ${ }^{19,29}$. Therefore, the decreased RVH and RV mass is consistent with the improved RV function we observed in SAB+MCT rats, as compared to MCT rats.

Second, SAB treatment also reduced RV fibrosis in MCT rats (Fig. 7A); although, this improvement did not alter RVEDP (Supplemental Fig. S3). Similar to our observation, a previous study showed that $\mathrm{SAB}$ reduced $\mathrm{RV}$ fibrosis in $\mathrm{PAB}$ rabbits, although this was associated with a small increase in $\mathrm{RVEDP}^{26}$. The reason for the concordant findings of reduced fibrosis but discordant findings in terms of RVEDP between the two studies is unknown, although both the species studied (rat vs. rabbit) and the intervention used to induce RVH (MCT vs. PAB) differed between studies. 
Third, SAB improved RV contractile function in MCT rats, as indicated by increased RVFW thickening, TAPSE and RV-PA coupling (Figs. 3A\&4). SAB also caused a slight decrease in RVSP (Supplemental Fig. S3). This is consistent with the previous study that treated the PAB model of RVH with SAB and showed it improved RV contractile function without altering RVSP ${ }^{26}$. SAB increases LV CO in MCT rats (Fig. 3C), similar to the effects seen when coronary perfusion pressure (aortic pressure) is increased using phenylephrine in adult dogs suffering from acute, PAB-induced RV failure ${ }^{25}$. In our study, measures of improved RV function (increased RVFW thickening, TAPSE, RV-PA coupling and LV CO) correlated directly with enhanced systolic but not with diastolic RCA-PP (Fig. 5). Note that decreased LV perfusion was observed in MCT rats (Fig. 6A), as has been reported in PAH patients. ${ }^{19}$ This probably reflects the lower CO that occurs in PAH. SAB partially restored LV perfusion in SAB+MCT rats (Fig. 6A). While the significance of the alteration of LV perfusion by MCT and SAB is unknown, SAB did not alter LV contractile function in MCT rats (Fig. 4C), reinforcing the selective importance of improving systolic RCA blood flow (but not LV function) in improving RV contractile function.

Surprisingly, SAB reduced PVR and increased PAAT in MCT rats (Fig. 3A, Supplemental Fig. S3). These finding suggest that pulmonary hypertension severity was reduced; however, there was no significant improvement by $\mathrm{SAB}$ in the pulmonary vasculature, evident as a lack of change in the muscularized pulmonary vessels or in the medial wall thickness/external diameter ratio (Fig. 7D). Since SAB increases aortic pressure and thus increases RCA-PP and possibly flow, RV function improves as a result. Our group has shown that SAB alone, albeit a more severe aortic constriction ( $60 \%$ constriction), in rats for 9 weeks actually causes adverse pulmonary vascular remodeling, leading to increased PVR and reduced $\mathrm{PAAT}^{31}$. In the current study, milder SAB ( 40\% constriction) for a shorter duration (4 weeks) did not exacerbate MCT pulmonary vascular 
remodeling or cause adverse PA remodeling on its own. However, the basis for the observed reduction in PVR and increase in PAAT in SAB+MCT rats seems to be related to improved CO. Vascular resistance is inversely proportional to the fourth power of vessel radius. Since CO was increased in $\mathrm{SAB}+\mathrm{MCT}$ rats due to improved $\mathrm{RV}$ contractile function, the cross-sectional area of pulmonary vasculature perfused presumably increased, leading to reduced PVR and increased PAAT.

In addition, MCT is known to cause a metabolic shift to uncoupled glycolysis in the $\mathrm{RV}^{12}$. We examined changes in the isoform expression of the terminal glycolytic enzyme, pyruvate kinase muscle isoforms 1 and 2, and PKM2/PKM1 ratio in RV. We did this because our prior work showed a shift favoring the proglycolytic isoform PKM2 occurs in the RV and LV in response to a more severe and sustained aortic constriction ${ }^{31}$. Moreover, an increase in the PKM2/PKM1 ratio has been shown to be associated with a shift toward aerobic glycolysis in other PAH cells, including PA endothelial cells ${ }^{32}$ and PA fibroblasts ${ }^{33}$. Consistent with this, we observed an increase in PKM2/PKM1 ratio in the MCT RVs (Fig. 8). Conversely, in SAB+MCT RVs, the PKM2/PKM1 ratio was reversed toward normal, consistent with a restoration of oxidative metabolism (Fig. 8). In addition, there was a trend toward a decrease in P-PDH/PDH in SAB+MCT vs. MCT RVs (Supplemental Fig. S5), also suggesting the restoration of oxidative metabolism. It is suspected that $\mathrm{SAB}$ improved RV function in MCT rats by preventing this glycolytic shift via restoring the PKM2/PKM1 and P-PDH/PDH ratios (Fig. 8).

Finally, MCT caused RV microvascular rarefaction (Fig. 7C), which is consistent to previous studies on MCT rats ${ }^{17,22}$, Sugen-hypoxia rats $^{18}$, and PAH patients ${ }^{22}$. However, SAB treatment did not improve the RV microvasculature in MCT rats (Fig. 7C). This suggests that the 
improved RV function by $\mathrm{SAB}$ is not due to the improvement in RV microvasculature but rather reflects improved epicardial RCA perfusion (Fig. 6).

Despite these benefits, SAB is presented as a proof of concept, rather than as a therapeutic strategy for application in patients with PAH. However, as a proof of concept, SAB demonstrates the benefits of maintaining coronary perfusion pressure by increasing systemic blood pressure while eliminating the confounding effects of increasing aortic pressure using vasoconstrictors, such as phenylephrine. There are lessons for patient management suggested by this study. Most systemic vasoconstrictor agents cause concomitant and harmful pulmonary vasoconstriction. For example, we might consider treating RV failure in $\mathrm{PAH}$ by increasing aortic pressure pharmacologically. This has been attempted in patients with chronic pulmonary hypertension who had anesthesia induced hypotension. Phenylephrine infusion did elevate systemic blood pressure but did not do so preferentially, resulting in a concomitant increase in PA pressure ${ }^{27,28}$. Pulmonary vasoconstriction would counteract the desired increase in RCA-PP one hopes to achieve. Our study also reinforces the well accepted advice against using systemic vasodilators in PAH patients, since these drugs would be predicted to reduce aortic root pressure, decrease RV perfusion and worsen RV function.

\section{Limitations}

We wish to highlight several limitations in this study. First, we only examined limited enzymes involved in glucose metabolism, PKM2, PKM1 and P-PDH, and did not directly measure RV metabolism. Nevertheless, our data are consistent with SAB improving RV function in MCT rats at least partially via restoring the PKM2/PKM1 ratio, presumably by enhancing RV bioenergetics. Second, the SAB surgery was performed before the injection of MCT, i.e., before 
the induction of PAH. Therefore, this is a prevention rather than a regression study. Third, we only used the MCT rat model because it is known to have the most severe RV dysfunction of the preclinical PAH models. Other PAH animal models such as the Sugen-hypoxia rat model should be tested in the future. Finally, we acknowledge this study is a proof of concept study that is not intended to reflect a therapeutic intervention that can easily be translated to the care of patients with PAH.

\section{Conclusions}

This study offers four important findings that illustrate the crucial contribution of RCA ischemia to the etiology of RV dysfunction in experimental PAH. First, PAH reduces RCA-PP in systole, and this reduces RV perfusion, which contributes to reduced RV function. Second, SAB (by increasing systolic RCA-PP) improves RV perfusion in MCT rats. Third, the benefits of improving epicardial RCA perfusion include not only improved RV function but also reduced RVH and RV fibrosis. Finally, SAB did not alter the RV microvasculature (i.e., capillary density) or pulmonary remodeling but did improve $\mathrm{CO}$ and thus enhanced perfusion of both the heart and lung. These findings highlight the importance of RCA perfusion for RV function and show that chronically increasing systolic RCA perfusion alone, without altering the RV microvasculature, can improve RV function in PAH. 


\section{Materials and Methods}

All the experimental protocols were approved by Queen's University Animal Care Committee and the University Research and Ethics Board.

\section{Statistical Analysis}

All the data are reported as mean \pm standard error of mean (SEM). Two-tailed, Student's $t$-test, paired $t$-test, regression analysis, or analysis of variance (ANOVA) was performed as appropriate. Statistical analyses were performed with the GraphPad Prism (GraphPad8 Software, La Jolla, CA, United States). A $P$ value of less than 0.05 was considered statistically significant. A linear regression analysis was performed between RVFW thickening and RCA-PP, between TAPSE and RCA-PP, between RV end-systolic elastance and RCA-PP, between CO and RCAPP, to evaluate the effects of RCA-PP on RV contractility. A linear regression analysis was also performed between RV-PA coupling estimated from single-beat method and from combined catheterization data (RVSP) and echocardiography data (RVFW thickening and TAPSE). Careful attention was made to blinding investigators to experimental groups. Rats were randomly assigned to treatment groups.

\section{Experimental Design}

SAB or sham surgery was performed on male Sprague Dawley rats (125-150 g) (Charles River, Montreal, QC, Canada) following the protocol in Fig. 1. 7-10 days after the surgery, these were randomized to receive a single subcutaneous injection of either phosphate-buffered saline (PBS; $2 \mathrm{~mL} / \mathrm{kg}$ ) or MCT (60 mg/kg). Rats were randomized to 4 experimental groups including Control, SAB, MCT, and SAB+MCT (n=10, 9, 14 and 14, respectively), in which Control and 
SAB groups were injected with PBS and Control and MCT groups had sham surgery before injection. Not all the animals underwent all these measurements: cardiac catheterization, echocardiography, and SPECT. For example, the sample size for rats that underwent both echocardiography and catheterization in Control, SAB, MCT and SAB+MCT groups was n=5, 6, 9 and 9 rats, respectively. The rats that underwent catheterization did not have SPECT study. The reasons that we did not perform all studies in all rats included the need to avoid excessive anesthesia duration, the limited duration of hemodynamic stability during heart catheterization, and a restriction on locations within the center where we can use SPECT imaging.

Cardiac function and structure (using high resolution echocardiography) and cardiac hemodynamics (using closed-chest, micromanometer-tipped catheters to perform cardiac catheterization) were measured after 4 weeks, as previously described ${ }^{34}$. RV perfusion and function were quantified applying SPECT sestamibi imaging technique with ${ }^{99 m}$ Tc-MIBI in an additional 3 rats/group. In several additional animals/group, RV perfusion was measured by imaging infused fluorescent microspheres using a super-resolution confocal microscope in a field of view 3-dimensional mode. After cardiac catheterization, hearts and lungs were harvested for weight measurement of RV and LV plus septum, RV histology and protein, and quantitative pulmonary vascular histology, as previously described ${ }^{31,35}$.

\section{SAB Surgery}

SAB surgery was performed as previously described ${ }^{31}$. Briefly, rats were anesthetized with isoflurane (2-2.5\%), intubated, mechanically ventilated (60-70 breath/minute, tidal volume of 6.0 $\mathrm{mL} / \mathrm{kg}$ ) and, using sterile technique, a $2 \mathrm{~cm}$ skin incision was made between the left $2^{\text {nd }}$ and $3^{\text {rd }}$ rib. After dissecting apart the intercoastal muscle, the ascending aorta was visualized and isolated. A 
3 x 3 mm titanium clip (Weck ${ }^{\circledR}$ Horizon $^{\mathrm{TM}}$ Catalog \#523735, Teleflex, Markham, ON, Canada) was placed around the ascending aorta $\sim 5 \mathrm{~mm}$ above the coronary arteries. This constricted the aorta by $\sim 40 \%$. The clip was applied with a modified applicator that has a calibrated stopper, so that upon full closure the applicator tips were uniformly $1.25 \mathrm{~mm}$ apart, thus yielding reproducible banding severity. The constriction for this study is less severe as compared to our prior study (in which we constricted the aorta by $\sim 60 \%$, applicator tips $\sim 1.0 \mathrm{~mm}$ apart $)^{31}$. Following aortic constriction, the lung was re-expanded by a chest tube and then the muscle layers and the skin were closed with interrupted 4-0 $\mathrm{Vicryl}^{\mathrm{TM}}$ sutures. For sham surgery, all the procedures were identical, except that no clip was placed around the ascending aorta. After surgery, standard postoperative care, including analgesia (buprenorphine $0.05 \mathrm{mg} / \mathrm{kg}$ ) was provided.

\section{Echocardiography}

At week 4 post injection, Doppler, 2-dimensional, and M-mode echocardiography was performed in rats anesthetized with isoflurane (2-2.5\%) using a high-frequency ultrasound system (Vevo 2100; Visual Sonics, Toronto, ON, Canada), as described ${ }^{21,34}$. Briefly, color Doppler guided pulsed-wave Doppler was used to guide pulsed Doppler measurement of PAAT and systolic velocity time integral (VTI) in the pulmonary outflow tract. Short axis view in B-mode was performed to obtain the main PA inner diameter (ID) during mid-systole. The parasternal long axis view was used to obtain M-mode measurements of the diastolic and systolic thickness of the RVFW. A modified apical four chamber view was used for M-mode measurement of TAPSE. RVFW systolic thickening was calculated as $\left(\mathrm{RVFW}_{\text {systole }}-\mathrm{RVFW}_{\text {diastole }}\right) / \mathrm{RVFW}_{\text {diastole }}$, and CO was estimated as HR $\mathrm{x}$ VTI $\mathrm{x} \pi \times \mathrm{ID}^{2} / 4$, where $\mathrm{HR}$ is the heart rate obtained from electrocardiography trace, as described previously ${ }^{12,21,34}$. 


\section{Cardiac catheterization}

Cardiac catheterization was performed as described ${ }^{31}$. Briefly, rats were anesthetized with isoflurane (2.5 to $3.0 \%$ ). Left (via right carotid artery) and right (via right jugular vein) heart catheterization (LHC and RHC, respectively) was performed in closed-chest rats using micromanometer, high-fidelity 1.9-F rat pressure-volume catheter (Transonic, London, ON, Canada). LVSP, LVEDP, aortic systolic and diastolic pressures, stroke volume, CO, and heart rate were obtained from LHC. RVSP and RVEDP were obtained from RHC. RCA-PP was calculated as (aortic pressure proximal to band-RV pressure). PVR was calculated as (RVSP-LVEDP)/CO.

\section{Ventricular-vascular coupling}

RV-PA coupling was assessed as TAPSE or RVFW thickening divided by RVSP, as previously described ${ }^{36,37}$. In addition, single-beat method was employed to estimate both LV and RV end-systolic elastance $\left(E_{e s}\right)$. Effective arterial elastance $\left(E_{a}\right)$ was calculated from LV or RV pressure-volume loops. Ventricular-vascular coupling is calculated as the ratio of $\mathrm{E}_{\mathrm{es}}$ to $\mathrm{E}_{\mathrm{a}}$, i.e., $\mathrm{E}_{\mathrm{es}} / \mathrm{E}_{\mathrm{a}}$. See supplemental methods for detailed implementation of single-beat method and the calculation of $\mathrm{E}_{\mathrm{a}}$.

\section{Histological Analysis}

After sacrificing the rats, fresh RV and LV were harvested and fixed in $10 \%$ buffered formalin. The left lobe of the lung was perfused with $10 \%$ formalin at $25 \mathrm{~cm} \mathrm{H}_{2} \mathrm{O}$ pressure for $\sim 30$ minutes, and then stored in formalin. Tissues were embedded in paraffin wax, sectioned, and stained with picrosirius red for collagen deposition or hematoxylin and eosin. See supplemental 
methods for details of imaging and quantitative histology, all of which were done in a blinded fashion.

\section{MicroCT/SPECT Imaging}

The rat ( $\mathrm{n}=3$ per group) was anesthetized using isoflurane in $1 \mathrm{~L} / \mathrm{min}$ oxygen $(4-4.5 \%$ isoflurane for induction, and $2-2.5 \%$ for maintaining during scan). For cardiac- and respiratorygated imaging, EKG-leads and a chest wall respiratory transducer pad were applied to generate the image acquisition time points using the BioVet software (m2m Imaging Corp., Cleveland, OH, USA). SPECT/CT images were acquired in supine rats 30 minutes after tail vein injection of approximately $110 \pm 15 \mathrm{MBq}$ technetium sestamibi $\left({ }^{99 \mathrm{~m}} \mathrm{Tc}-\mathrm{MIBI}\right)$ using VECTor ${ }^{4} \mathrm{CT}$ pre-clinical scanner (MILabs B.V., Utrecht, Netherlands) equipped with multi-pinhole collimators and a conebeam X-ray CT system ${ }^{38}$. For this study, SPECT scans were acquired in an ultra-focus mode with a high-energy ultra-high-resolution rat/mouse pinhole collimator (HE-UHR-RM - $1.8 \mathrm{~mm}$ pinholes).

Scan volume or region of interest (ROI) was determined by using optical camera images followed by taking several two-dimensional X-ray cone-beam projections to make sure the entire heart was scanned. After a fast CT scan $(55 \mathrm{kVp}, 0.33 \mathrm{~mA}$ and 180 projections/rotation, $20 \mathrm{~ms}$ exposure time) for calculating attenuation and scatter corrections, a multi-frame SPECT acquisition was performed consisting of 4 subsequent, 10-minute scans.

\section{MicroCT/SPECT image reconstruction and analysis}

Image reconstructions were carried out using the Similarity-Regulated Ordered Subsets Expectation Maximization (SROSEM) algorithm. This algorithm includes resolution recovery and 
compensation for distance-dependent pinhole sensitivity ${ }^{39}$. For the SPECT images, we used 128 subsets and 5 iteration image reconstruction with an isotropic $0.6 \mathrm{~mm}$-voxel grid. Reconstructed SPECT images were quantified to correct for attenuation and scatter, using the CT image as an attenuation map for non-uniform attenuation correction. Additionally, ${ }^{99 \mathrm{~m}} \mathrm{Tc}-\mathrm{MIBI}$ signals were corrected for isotope decay (half-life $=6$ hours).

For quantification, the registered CT and quantified SPECT images were analyzed using PMOD 3.9 software (PMOD Technologies Ltd., Zurich, Switzerland) allowing short axis (SA), horizontal long axis (HLA) and vertical long axis (VLA) slices to be displayed. PMOD software was subsequently used for all quantification analyses (Supplementary Fig. S6). Two-dimensional ROIs were manually drawn to identify the radioactivity uptake within the RV and LV myocardium. Normalized uptakes, relative to the background radioactivity uptake, were calculated for each ROI in diastolic and systolic slices. Radioactivity concentration $(\mathrm{MBq} / \mathrm{cc})$ was corrected for the injected dose per gram of body weight.

\section{Microsphere injection, imaging and analysis}

The rat was anesthetized ( $3 \%$ isoflurane), intubated and ventilated $[\mathrm{n}=2$ per group (Control, MCT, SAB+MCT)]. The chest was opened by a sternotomy and the LV apex was localized. Dragon green microspheres (4.19 $\mu \mathrm{m}$ in diameter, FSDG006, Band Laboratories; Fishers, IN, USA) were injected via a 20-gauge needle inserted into the LV apex at a dose of $0.1 \mathrm{~mL} / 300 \mathrm{~g}$ body weight $\left(2.38 \times 10^{7}\right.$ microspheres/300 g). One minute after injection, both atria, the ascending aorta, and the main pulmonary artery were occluded with suture, and the whole heart was then isolated and dissected. After placing the heart in a tissue slicing matrix (Acrylic Brain Matrix, Adult Rat, \#15054; Ted Pella, Redding, CA, USA), a transverse section (2 mm in thickness) was obtained by 
cutting the heart with two razor blades simultaneously. The blood outside tissue of the transverse section was carefully washed out. The transverse section of the heart (with distinct RV and LV chambers) was then placed into a 35 mm glass bottom dish (P35G-1.5-14-C; MatTek, Ashland, MA, USA) and imaged using the Leica TCS SP8 confocal microscope (Leica Microsystems, Wetzlar, Germany). A white light laser system was adjusted to image the heart at $491 \mathrm{~nm}$ (the excitation of fluorescein isothiocyanate, FITC) and the signal was captured using the Leica HyD hybrid detectors. Images were taken in a z-stack (10 $\mu \mathrm{m}$ in $\mathrm{z}$ dimension $)$ and field of view tile scan mode. A total of 128 to 336 tiles per heart section were taken and merged to obtain the image of the whole RV (68-107 $\mathrm{mm}^{2}$, depending on the size of each heart). Images were captured using the HC PL APO 0.80NA, 20x dry objetive and analyzed with the LAS X Leica software. Maximum projection of the images was used to quantify the microspheres. Three or more ROIs were randomly drawn in each RV by a microscopist blinded to the treatment groups and the number of microspheres were quantified and normalized by the area of ROI and averaged for each RV.

\section{Immunofluorescence}

The RV microvasculature was stained for CD31 (ab182981, Abcam; Cambridge, MA, USA), as previously described ${ }^{40}$ (also see Supplemental methods). The capillary density in the RV was assessed by manually counting the number of microvessels $(1-10 \mu \mathrm{m}$ in diameter) per field $(184 \mu \mathrm{m} \times 184 \mu \mathrm{m})$.

\section{Immunoblotting}

Protein was extracted from frozen RV tissues and 40-60 $\mu \mathrm{g}$ of total protein was loaded to SDS-PAGE gel for immunoblot analysis, as previously described ${ }^{31}$ (also see Supplemental 
methods). The following antibodies were used: PDH E1-alpha subunit (phospho S293) (P-PDH) (ab177461; Abcam, Cambridge, MA, USA), PDH E1- $\alpha$ subunit (ab110334; Abcam, Cambridge, MA, USA), PKM1 (NBP2-14833; Novus Biologicals, Littleton, CO, USA), PKM2 (60268-1-Ig; Proteintech, Rosemont, IL, USA), and vinculin (V9131; Sigma, St. Louis, MO, USA). Vinculin was used as loading control. 


\section{Acknowledgements}

The authors wish to thank Ms Monica Neuber-Hess for her expert assistance with training in rodent surgery and for technical set-up of the surgical suite.

\section{Conflict of Interest}

None

\section{Sources of Funding}

This study is supported in part by U.S. National Institutes of Health (NIH) grants NIH R01HL113003 (S.L.A.) and NIH R01HL071115 (S.L.A), Canada Foundation for Innovation 229252 and 33012 (S.L.A.), Tier 1 Canada Research Chair in Mitochondrial Dynamics and Translational Medicine 950-229252 (S.L.A.), the William J. Henderson Foundation (S.L.A.), Canadian Vascular Network Scholar Award (L.T., F.P.), the Paroian Family PHA Canada scholarship (F.P.), funding from Queen's Clinician Investigator Program (P.Y. X.), Franklin Bracken Fellowship (P.Y.X.), and Susan Elizabeth Philips scholarship (P.Y.X.). This research is supported by Queen's CardioPulmonary Unit (QCPU). 


\section{References}

1. Thenappan T, Ormiston ML, Ryan JJ and Archer SL. Pulmonary arterial hypertension: pathogenesis and clinical management. BMJ. 2018;360:j5492.

2. Sandoval J, Bauerle O, Palomar A, Gomez A, Martinez-Guerra ML, Beltran M and Guerrero ML. Survival in primary pulmonary hypertension. Validation of a prognostic equation. Circulation. 1994;89:1733-44.

3. D'Alonzo GE, Barst RJ, Ayres SM, Bergofsky EH, Brundage BH, Detre KM, Fishman AP, Goldring RM, Groves BM, Kernis JT and et al. Survival in patients with primary pulmonary hypertension. Results from a national prospective registry. Ann Intern Med. 1991;115:343-9.

4. Campo A, Mathai SC, Le Pavec J, Zaiman AL, Hummers LK, Boyce D, Housten T, Champion HC, Lechtzin N, Wigley FM, Girgis RE and Hassoun PM. Hemodynamic predictors of survival in scleroderma-related pulmonary arterial hypertension. Am J Respir Crit Care Med. 2010;182:25260.

5. Ghio S, Klersy C, Magrini G, D'Armini AM, Scelsi L, Raineri C, Pasotti M, Serio A, Campana $\mathrm{C}$ and Vigano $\mathrm{M}$. Prognostic relevance of the echocardiographic assessment of right ventricular function in patients with idiopathic pulmonary arterial hypertension. International journal of cardiology. 2010;140:272-8.

6. Humbert M, Sitbon O, Chaouat A, Bertocchi M, Habib G, Gressin V, Yaici A, Weitzenblum E, Cordier JF, Chabot F, Dromer C, Pison C, Reynaud-Gaubert M, Haloun A, Laurent M, Hachulla E, Cottin V, Degano B, Jais X, Montani D, Souza R and Simonneau G. Survival in patients with idiopathic, familial, and anorexigen-associated pulmonary arterial hypertension in the modern management era. Circulation. 2010;122:156-63.

7. Sachdev A, Villarraga HR, Frantz RP, McGoon MD, Hsiao JF, Maalouf JF, Ammash NM, McCully RB, Miller FA, Pellikka PA, Oh JK and Kane GC. Right ventricular strain for prediction of survival in patients with pulmonary arterial hypertension. Chest. 2011;139:1299-1309.

8. Voelkel NF, Bogaard HJ and Gomez-Arroyo J. The need to recognize the pulmonary circulation and the right ventricle as an integrated functional unit: facts and hypotheses (2013 Grover Conference series). Pulmonary circulation. 2015;5:81-9.

9. van Wolferen SA, Marcus JT, Boonstra A, Marques KM, Bronzwaer JG, Spreeuwenberg MD, Postmus PE and Vonk-Noordegraaf A. Prognostic value of right ventricular mass, volume, and function in idiopathic pulmonary arterial hypertension. Eur Heart J. 2007;28:1250-7.

10. Basu S, Alzeair S, Li G, Dadparvar S and Alavi A. Etiopathologies associated with intercostal muscle hypermetabolism and prominent right ventricle visualization on 2-deoxy-2[F-18]fluoroD-glucose-positron emission tomography: significance of an incidental finding and in the setting of a known pulmonary disease. Mol Imaging Biol. 2007;9:333-9.

11. Oikawa M, Kagaya Y, Otani H, Sakuma M, Demachi J, Suzuki J, Takahashi T, Nawata J, Ido T, Watanabe J and Shirato K. Increased [18F]fluorodeoxyglucose accumulation in right ventricular free wall in patients with pulmonary hypertension and the effect of epoprostenol. J Am Coll Cardiol. 2005;45:1849-55.

12. Piao L, Fang YH, Cadete VJ, Wietholt C, Urboniene D, Toth PT, Marsboom G, Zhang HJ, Haber I, Rehman J, Lopaschuk GD and Archer SL. The inhibition of pyruvate dehydrogenase kinase improves impaired cardiac function and electrical remodeling in two models of right ventricular 
hypertrophy: resuscitating the hibernating right ventricle. Journal of molecular medicine. 2010;88:47-60.

13. Potus F, Hindmarch CCT, Dunham-Snary KJ, Stafford J and Archer SL. Transcriptomic Signature of Right Ventricular Failure in Experimental Pulmonary Arterial Hypertension: Deep Sequencing Demonstrates Mitochondrial, Fibrotic, Inflammatory and Angiogenic Abnormalities. International journal of molecular sciences. 2018;19 (9): E2730.

14. Sutendra $G$ and Michelakis ED. Pulmonary arterial hypertension: challenges in translational research and a vision for change. Sci Transl Med. 2013;5:208sr5.

15. Ryan JJ and Archer SL. The right ventricle in pulmonary arterial hypertension: disorders of metabolism, angiogenesis and adrenergic signaling in right ventricular failure. Circulation research. 2014;115:176-88.

16. van Wolferen SA, Marcus JT, Westerhof N, Spreeuwenberg MD, Marques KM, Bronzwaer JG, Henkens IR, Gan CT, Boonstra A, Postmus PE and Vonk-Noordegraaf A. Right coronary artery flow impairment in patients with pulmonary hypertension. Eur Heart J. 2008;29:120-7.

17. Archer SL, Fang YH, Ryan JJ and Piao L. Metabolism and bioenergetics in the right ventricle and pulmonary vasculature in pulmonary hypertension. Pulmonary circulation. 2013;3:144-52.

18. Bogaard HJ, Natarajan R, Henderson SC, Long CS, Kraskauskas D, Smithson L, Ockaili R, McCord JM and Voelkel NF. Chronic pulmonary artery pressure elevation is insufficient to explain right heart failure. Circulation. 2009;120:1951-60.

19. Vogel-Claussen J, Skrok J, Shehata ML, Singh S, Sibley CT, Boyce DM, Lechtzin N, Girgis RE, Mathai SC, Goldstein TA, Zheng J, Lima JA, Bluemke DA and Hassoun PM. Right and left ventricular myocardial perfusion reserves correlate with right ventricular function and pulmonary hemodynamics in patients with pulmonary arterial hypertension. Radiology. 2011;258:119-27.

20. Torbicki A, Kurzyna M, Kuca P, Fijalkowska A, Sikora J, Florczyk M, Pruszczyk P, Burakowski $\mathrm{J}$ and Wawrzynska L. Detectable serum cardiac troponin $\mathrm{T}$ as a marker of poor prognosis among patients with chronic precapillary pulmonary hypertension. Circulation. 2003;108:844-8.

21. Tian L, Neuber-Hess M, Mewburn J, Dasgupta A, Dunham-Snary K, Wu D, Chen KH, Hong Z, Sharp WW, Kutty S and Archer SL. Ischemia-induced Drp1 and Fis1-mediated mitochondrial fission and right ventricular dysfunction in pulmonary hypertension. Journal of molecular medicine. 2017;95:381-393.

22. Piao L, Fang YH, Parikh K, Ryan JJ, Toth PT and Archer SL. Cardiac glutaminolysis: a maladaptive cancer metabolism pathway in the right ventricle in pulmonary hypertension. Journal of molecular medicine. 2013;91:1185-97.

23. Frump AL, Bonnet S, de Jesus Perez VA and Lahm T. Emerging role of angiogenesis in adaptive and maladaptive right ventricular remodeling in pulmonary hypertension. Am J Physiol Lung Cell Mol Physiol. 2018;314:L443-L460.

24. Westerhof BE, Saouti N, van der Laarse WJ, Westerhof N and Vonk Noordegraaf A. Treatment strategies for the right heart in pulmonary hypertension. Cardiovasc Res. 2017;113:1465-1473.

25. Vlahakes GJ, Turley $\mathrm{K}$ and Hoffman JI. The pathophysiology of failure in acute right ventricular hypertension: hemodynamic and biochemical correlations. Circulation. 1981;63:8795.

26. Apitz C, Honjo O, Humpl T, Li J, Assad RS, Cho MY, Hong J, Friedberg MK and Redington AN. Biventricular structural and functional responses to aortic constriction in a rabbit model of 
chronic right ventricular pressure overload. The Journal of thoracic and cardiovascular surgery. 2012;144:1494-501.

27. Rich S, Gubin S and Hart K. The effects of phenylephrine on right ventricular performance in patients with pulmonary hypertension. Chest. 1990;98:1102-6.

28. Kwak YL, Lee CS, Park YH and Hong YW. The effect of phenylephrine and norepinephrine in patients with chronic pulmonary hypertension*. Anaesthesia. 2002;57:9-14.

29. Crystal GJ and Pagel PS. Right Ventricular Perfusion: Physiology and Clinical Implications. Anesthesiology. 2018;128:202-218.

30. Bian X, Williams AG, Jr., Gwirtz PA and Downey HF. Right coronary autoregulation in conscious, chronically instrumented dogs. Am J Physiol. 1998;275:H169-75.

31. Xiong PY, Tian L, Dunham-Snary KJ, Chen KH, Mewburn JD, Neuber-Hess M, Martin A, Dasgupta A, Potus F and Archer SL. Biventricular Increases in Mitochondrial Fission Mediator (MiD51) and Proglycolytic Pyruvate Kinase (PKM2) Isoform in Experimental Group 2 Pulmonary Hypertension-Novel Mitochondrial Abnormalities. Front Cardiovasc Med. 2018;5:195.

32. Caruso P, Dunmore BJ, Schlosser K, Schoors S, Dos Santos C, Perez-Iratxeta C, Lavoie JR, Zhang $\mathrm{H}$, Long L, Flockton AR, Frid MG, Upton PD, D'Alessandro A, Hadinnapola C, Kiskin FN, Taha M, Hurst LA, Ormiston ML, Hata A, Stenmark KR, Carmeliet P, Stewart DJ and Morrell NW. Identification of MicroRNA-124 as a Major Regulator of Enhanced Endothelial Cell Glycolysis in Pulmonary Arterial Hypertension via PTBP1 (Polypyrimidine Tract Binding Protein) and Pyruvate Kinase M2. Circulation. 2017;136:2451-2467.

33. Zhang $\mathrm{H}$, Wang D, Li M, Plecita-Hlavata L, D'Alessandro A, Tauber J, Riddle S, Kumar S, Flockton A, McKeon BA, Frid MG, Reisz JA, Caruso P, El Kasmi KC, Jezek P, Morrell NW, Hu CJ and Stenmark KR. Metabolic and Proliferative State of Vascular Adventitial Fibroblasts in Pulmonary Hypertension Is Regulated Through a MicroRNA-124/PTBP1 (Polypyrimidine Tract Binding Protein 1)/Pyruvate Kinase Muscle Axis. Circulation. 2017;136:2468-2485.

34. Urboniene D, Haber I, Fang YH, Thenappan T and Archer SL. Validation of high-resolution echocardiography and magnetic resonance imaging vs. high-fidelity catheterization in experimental pulmonary hypertension. Am J Physiol Lung Cell Mol Physiol. 2010;299:L401-12.

35. Chen KH, Dasgupta A, Lin J, Potus F, Bonnet S, Iremonger J, Fu J, Mewburn J, Wu D, Dunham-Snary K, Theilmann AL, Jing ZC, Hindmarch C, Ormiston ML, Lawrie A and Archer SL. Epigenetic Dysregulation of the Drp1 Binding Partners MiD49 and MiD51 Increases Mitotic Mitochondrial Fission and Promotes Pulmonary Arterial Hypertension: Mechanistic and Therapeutic Implications. Circulation. 2018; 138(3):287-304.

36. Egbe AC, Kothapalli S, Miranda WR, Pislaru S, Ammash NM, Borlaug BA, Pellikka PA, Najam $\mathrm{M}$ and Connolly HM. Assessment of Right Ventricular-Pulmonary Arterial Coupling in Chronic Pulmonary Regurgitation. Can J Cardiol. 2019;35:914-922.

37. Prins KW, Tian L, Wu D, Thenappan T, Metzger JM and Archer SL. Colchicine Depolymerizes Microtubules, Increases Junctophilin-2, and Improves Right Ventricular Function in Experimental Pulmonary Arterial Hypertension. Journal of the American Heart Association. 2017;6.

38. Ivashchenko O, van der Have F, Villena JL, Groen HC, Ramakers RM, Weinans HH and Beekman FJ. Quarter-millimeter-resolution molecular mouse imaging with U-SPECT ${ }^{+}$. Molecular imaging. 2014;13. 
39. Vaissier P, Goorden M and Beekman FJ. Similarity-Regulated OSEM reconstruction for pinhole-PET. The Journal of Nuclear Medicine. 2015;56:48.

40. Hong Z, Chen KH, DasGupta A, Potus F, Dunham-Snary K, Bonnet S, Tian L, Fu J, BreuilsBonnet S, Provencher S, Wu D, Mewburn J, Ormiston ML and Archer SL. MicroRNA-138 and MicroRNA-25 Down-regulate Mitochondrial Calcium Uniporter, Causing the Pulmonary Arterial Hypertension Cancer Phenotype. Am J Respir Crit Care Med. 2017;195:515-529. 


\section{Legends to Figures}

Figure 1. Experimental design for the supra-coronary aortic banding (SAB) in monocrotaline (MCT) rat study. SD, Sprague-Dawley; SC: subcutaneous; PBS, phosphate-buffered saline; SPECT: single-photon emission computed tomography.

Figure 2. Supra-coronary aortic banding (SAB) reduces right ventricular (RV) hypertrophy (i.e., $\mathrm{RV}$ weight) in $\mathrm{SAB}+$ monocrotaline $(\mathrm{MCT})$ vs. MCT rats. $\mathrm{LV}+\mathrm{S}$, left ventricle plus septum. $†$, $\mathrm{P}<0.01$ and $\ddagger, \mathrm{P}<0.001$ for $\mathrm{SAB}$ vs. Control, MCT vs. Control, or SAB+MCT vs. SAB unless otherwise specified.

Figure 3. Supra-coronary aortic banding (SAB) reduces right ventricular free wall (RVFW) thickness and increases RVFW thickening, tricuspid annular plane systolic excursion (TAPSE), pulmonary artery acceleration time (PAAT), systolic right coronary artery perfusion pressure (RCA-PP) and left ventricular (LV) cardiac output (CO) in monocrotaline (MCT) rats in $\mathrm{SAB}+$ monocrotaline (MCT) vs. MCT rats. (A) Representative echocardiographic images of parasternal long-axis view of RVFW in M-mode, tricuspid annulus in M-mode, and pulmonary artery Doppler flow profile and summary of the data; (B) Representative traces of pressures in right ventricle (RV) and aorta proximal to the band; (C) Summary of RCA-PP and LV CO. *, P < $0.05, \uparrow, \mathrm{P}<0.01$ and $\ddagger, \mathrm{P}<0.001$ for $\mathrm{SAB}$ vs. Control, MCT vs. Control, or SAB+MCT vs. SAB unless otherwise specified.

Figure 4. Supra-coronary aortic banding $(\mathrm{SAB})$ improves right ventricle-pulmonary artery (RVPA) coupling in SAB+monocrotaline (MCT) vs. MCT rats. (A) Summary of indices for RV-PA 
coupling, i.e., RV free wall (RVFW) thickening/RV systolic pressure (RVSP) and tricuspid annular plane systolic excursion (TAPSE)/RVSP; (B) Summary of RV end-systolic elastance ( $\mathrm{E}_{\mathrm{es}}$ ), pulmonary effective arterial elastance $\left(\mathrm{E}_{\mathrm{a}}\right)$ and RV $\mathrm{E}_{\mathrm{es}} / \mathrm{E}_{\mathrm{a}}$; (C) Summary of LV $\mathrm{E}_{\mathrm{es}}$, systemic $\mathrm{E}_{\mathrm{a}}$ and $L V E_{e s} / E_{a} \cdot{ }^{*}, \mathrm{P}<0.05, \dagger, \mathrm{P}<0.01$ and $\ddagger, \mathrm{P}<0.001$ for MCT vs. Control or SAB+MCT vs. SAB unless otherwise specified.

Figure 5. Systolic (but not diastolic) right coronary artery perfusion pressure (RCA-PP) is well correlated with right ventricular free wall (RVFW) thickening, tricuspid annular plane systolic excursion (TAPSE), RV end-systolic elastance ( $\left.\mathrm{E}_{\mathrm{es}}\right)$, and left ventricular (LV) cardiac output (CO) in all the monocrotaline (MCT)-injected rats from MCT and supra-coronary aortic banding (SAB)+MCT groups, as indicated by linear regression analysis between RVFW thickening and RCA-PP, between TAPSE and RCA-PP, between RV E es and RCA-PP, and between LV CO and RCA-PP.

Figure 6. Supra-coronary aortic banding (SAB) tends to increase uptake of ${ }^{99 \mathrm{~m}} \mathrm{Tc}-\mathrm{MIBI}$ in both free walls of right ventricle (RV) and left ventricle (LV) at both systole and diastole and the density of microspheres in $\mathrm{RV}$ in $\mathrm{SAB}+$ monocrotaline (MCT) vs. MCT rats. (A) Representative short-axis view of heart showing the uptake of ${ }^{99} \mathrm{~m}$ Tc-MIBI during systole and diastole obtained by Singlephoton emission computed tomography (SPECT) technique and summary of the ratio of radioactivity $\left({ }^{99 \mathrm{~m}} \mathrm{Tc}-\mathrm{MIBI}\right)$ uptake during systole and diastole over their own background radioactivity, respectively. Standardized radioactivity uptake scale on the right: red represents relatively more uptake, whereas blue means relatively less uptake; (B) Correlation between RV cardiac output (CO) estimated from echocardiography and RV radioactivity uptake at systole and 
diastole. Note that we were unable to obtain hemodynamic data from all rats and thus this Figure includes data only from the following: 1 Control rat $(\mathrm{CO}=121 \mathrm{~mL} / \mathrm{min}), 1 \mathrm{SAB}$ rat $(\mathrm{CO}=96$ $\mathrm{mL} / \mathrm{min}$ ), $2 \mathrm{MCT}$ rats and $3 \mathrm{SAB}+\mathrm{MCT}$ rats; (C) Representative images of microspheres (green) in RV free wall (RVFW); (D) Summary of the number of microspheres in RVFW per $\mathrm{mm}^{2}$. *, $\mathrm{P}<$ $0.05, \uparrow, \mathrm{P}<0.01$ and $\ddagger, \mathrm{P}<0.001$ for $\mathrm{SAB}$ vs. Control, MCT vs. Control or SAB+MCT vs. SAB.

Figure 7. Supra-coronary aortic banding (SAB) reduces fibrosis and myocyte size in right ventricle (RV), but does not change capillary density in RV or muscularized pulmonary vessels or medial wall thickness in the lung in SAB+monocrotaline (MCT) vs. MCT rats. (A) Representative images of RVs stained with picrosirius red and summary of collagen area fraction; (B) Representative images of RVs stained with hematoxylin and eosin and summary of myocyte area; (C) Representative immunofluorescent images of RVs stained with CD31 and summary of the density of RV microvessels (i.e., CD31); (D) Representative images of lungs stained with picrosirius red at 40X (upper panels) and 5X (lower panels) fields and summary of the number of muscularized pulmonary vessels per $4.9 \mathrm{~mm}^{2}$ and the medial wall thickness to external diameter ratio. The $40 \mathrm{X}$ field (upper panels) shows the typical nonmuscularized vessels (in Control and $\mathrm{SAB}$ groups) and muscularized pulmonary vessels (in MCT and SAB+MCT groups). *, $\mathrm{P}<0.05$, $\dagger, \mathrm{P}<0.01$ and $\ddagger, \mathrm{P}<0.001$ for MCT vs. Control or SAB+MCT vs. SAB unless otherwise specified.

Figure 8. Supra-coronary aortic banding (SAB) restored pyruvate kinase muscle isoform 1 (PKM1) and PKM2/PKM1 ratio in the right ventricle (RV) in SAB+monocrotaline (MCT) vs. MCT rats. Representative western blot images and the summary of PKM2/PKM1 ratio are shown. $\uparrow, \mathrm{P}<0.01$ and $\ddagger, \mathrm{P}<0.001$ for MCT vs. Control unless otherwise specified. 


\section{Figure 1. Experimental design}

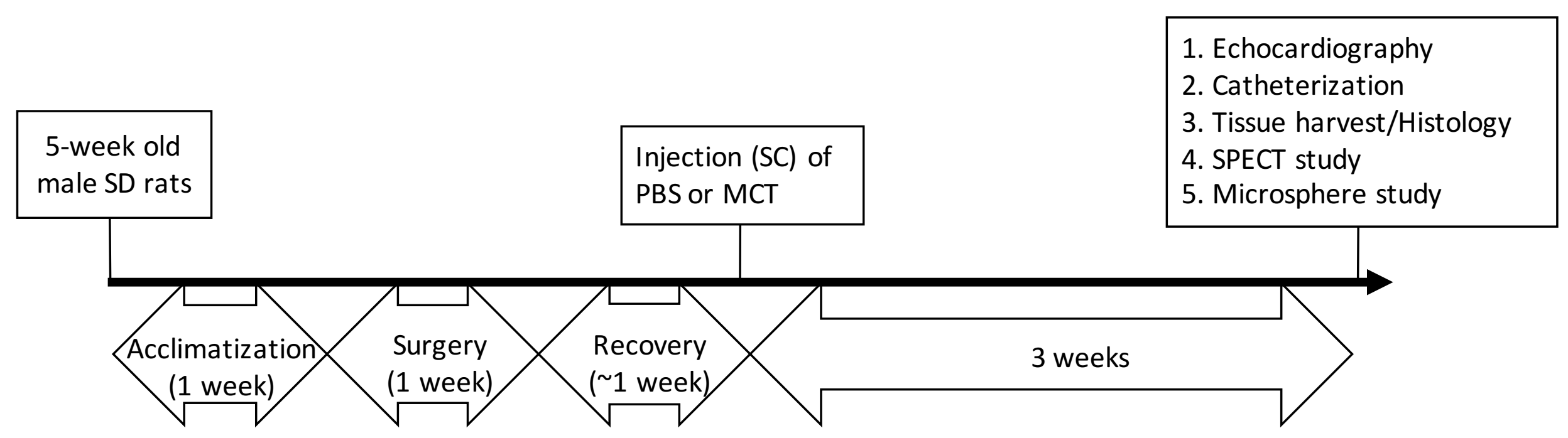


Figure 2. Body and heart weights
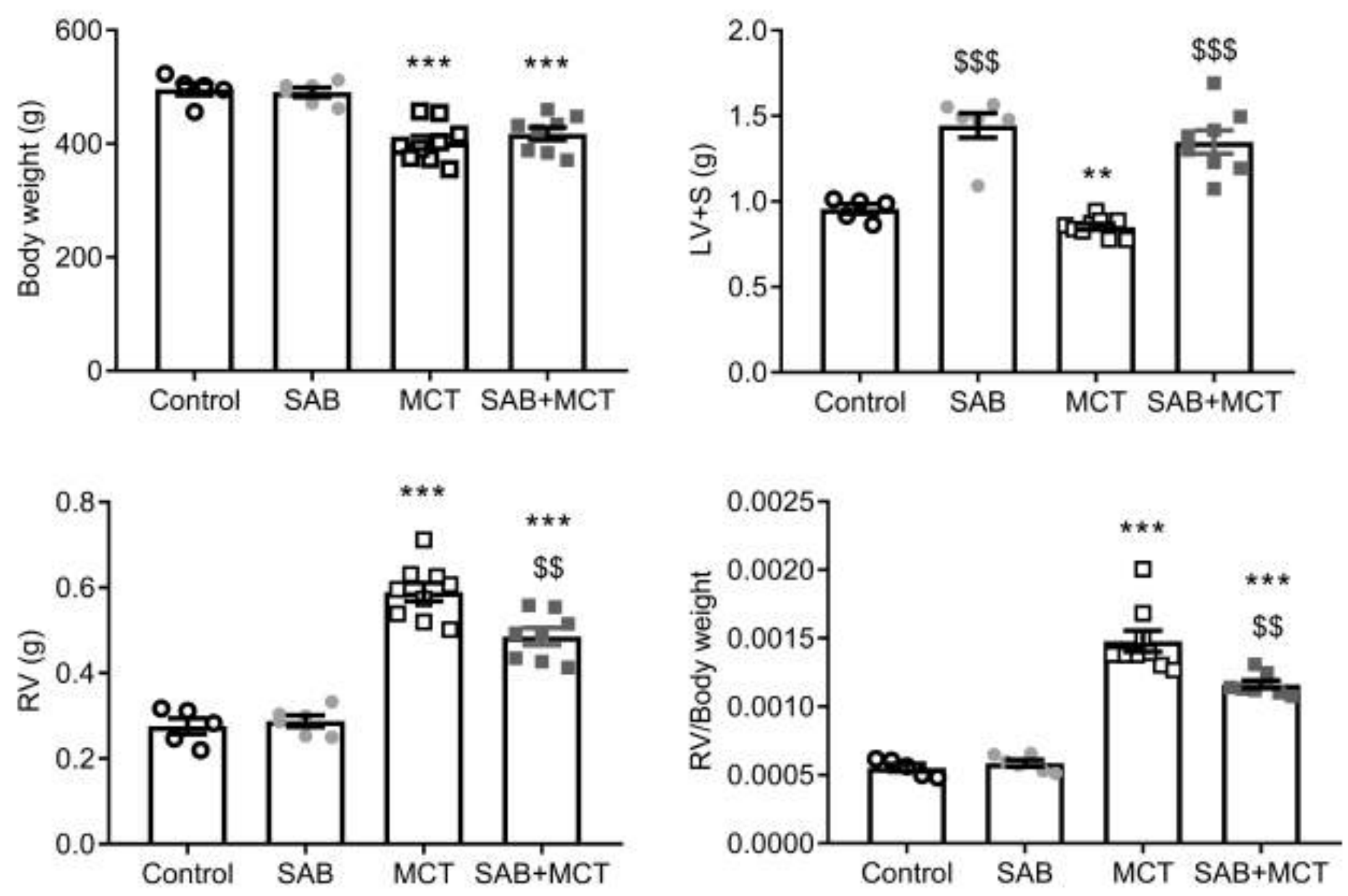
Figure 3. (A) Echo data

A

RVFW
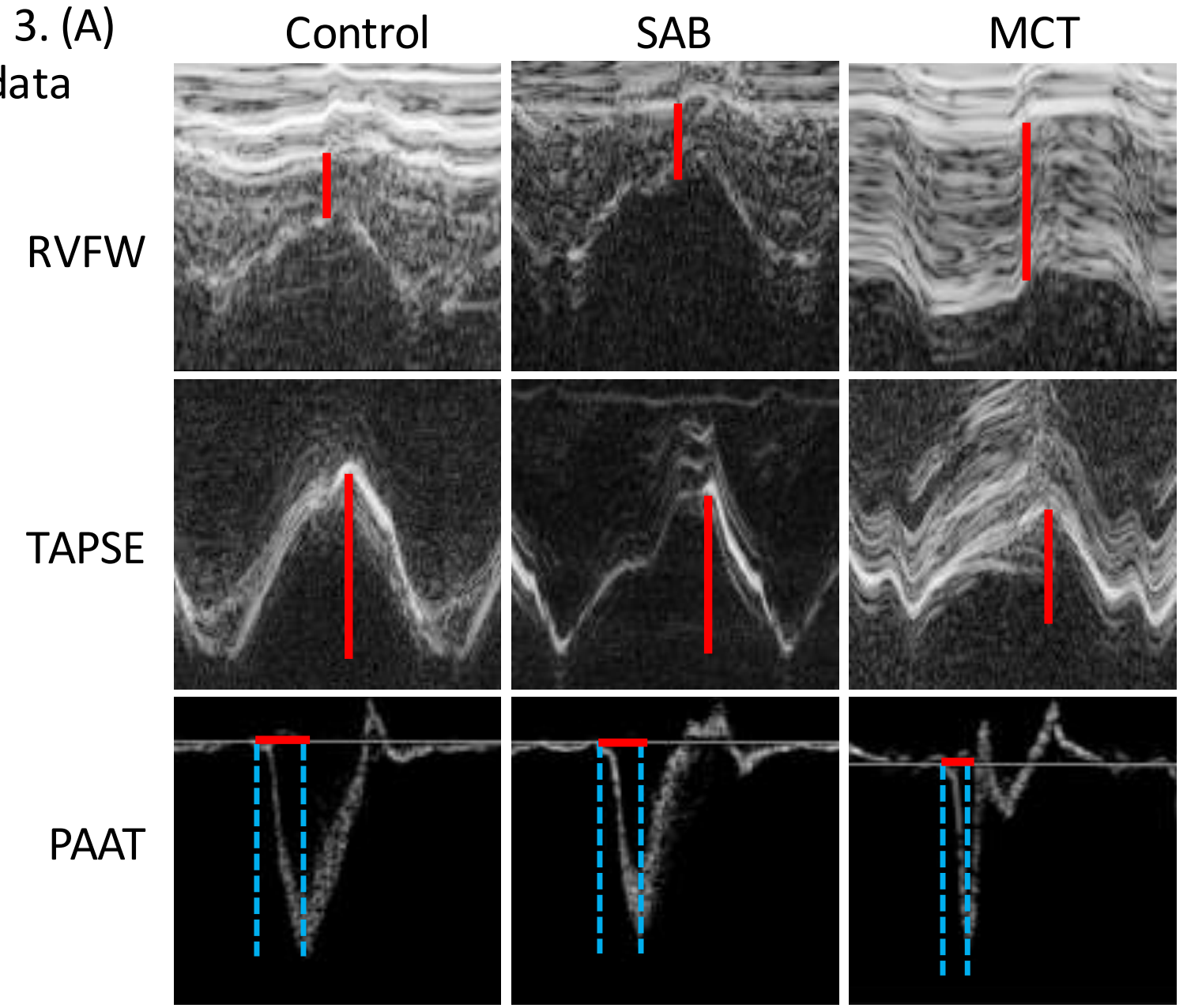

$S A B+M C T$
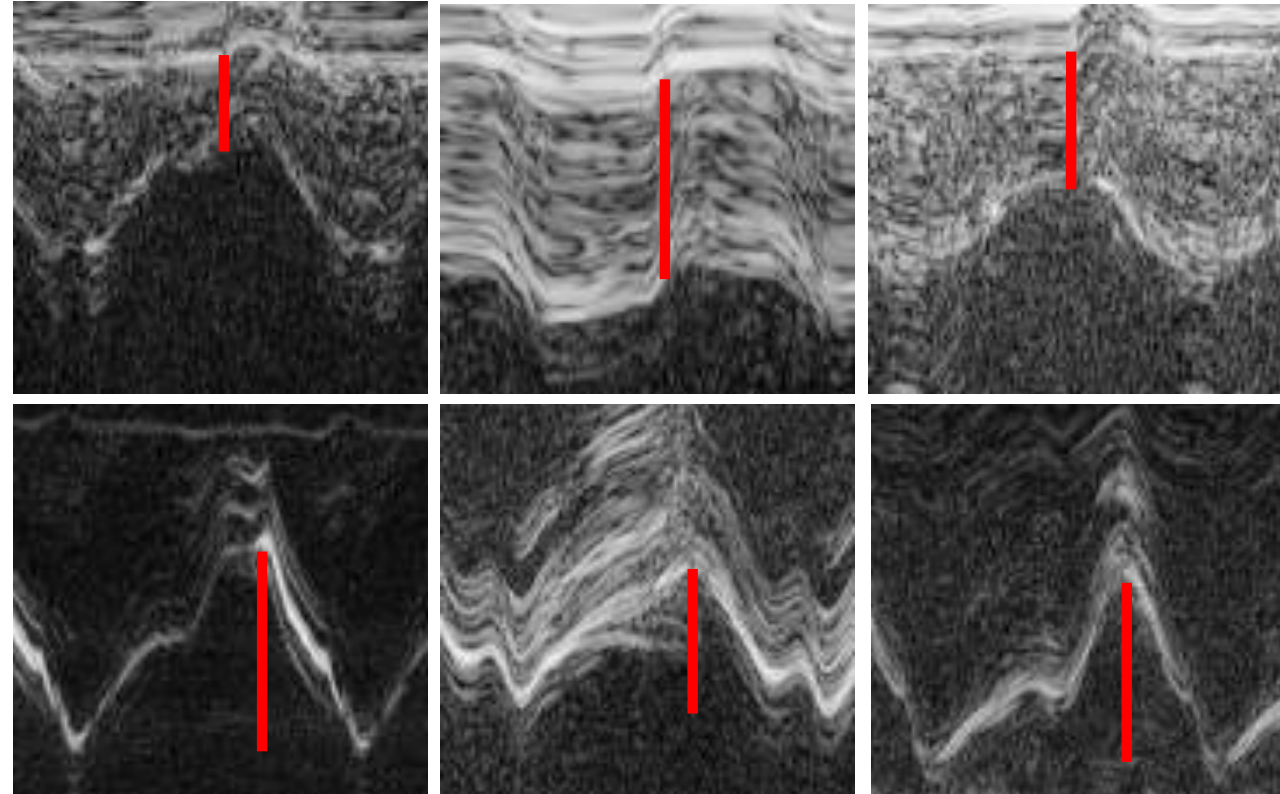

$3 \mathrm{~mm}$
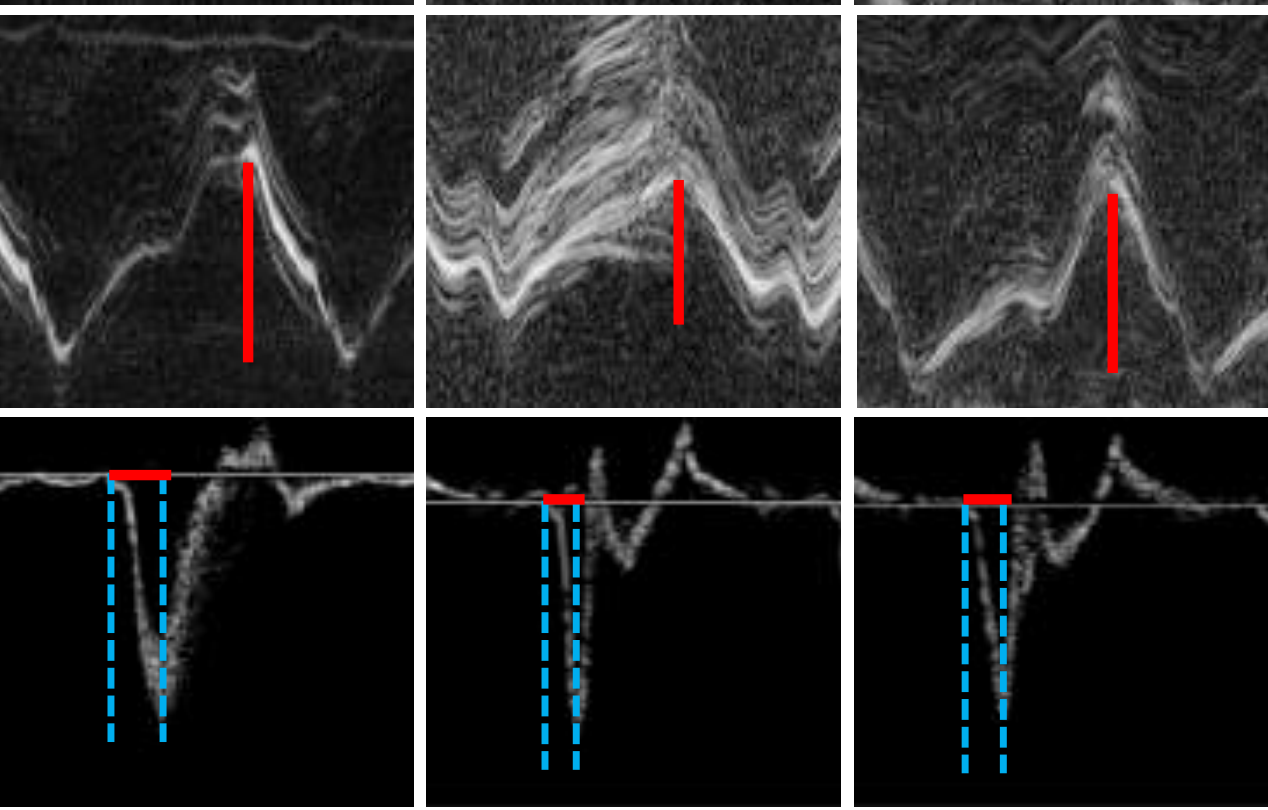

$5 \mathrm{~mm}$
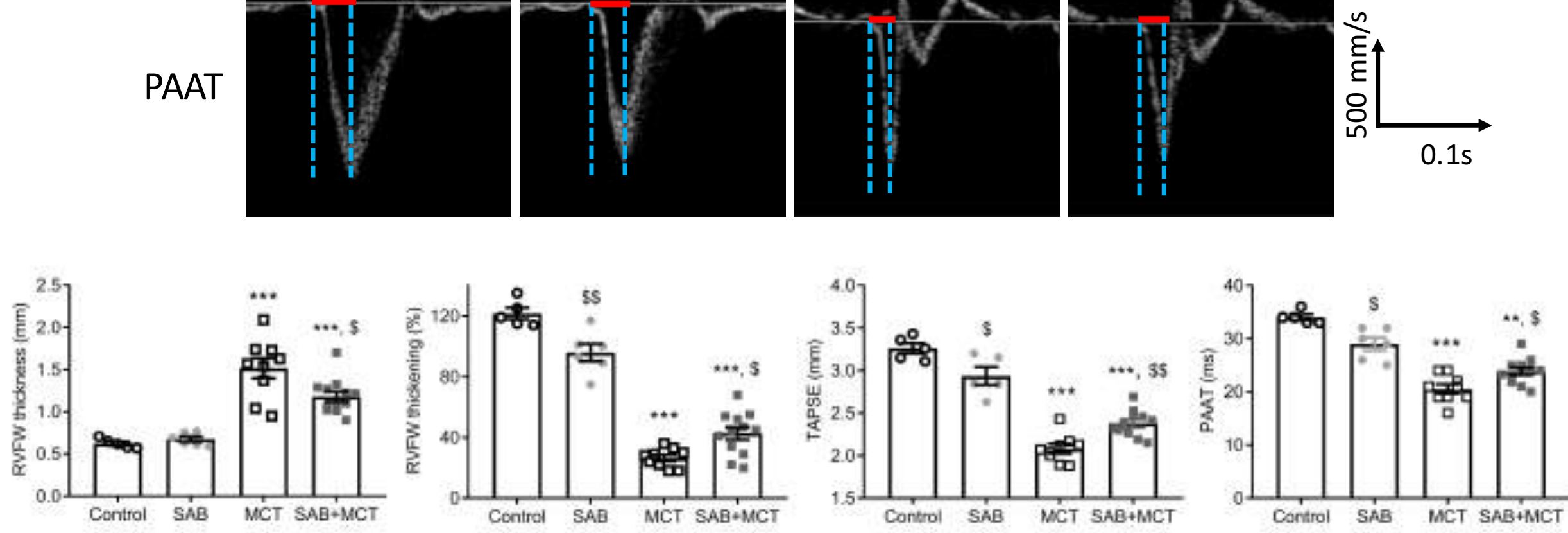
Figure 3.(B\&C) Hemodynamic data from cardiac catheterization

B
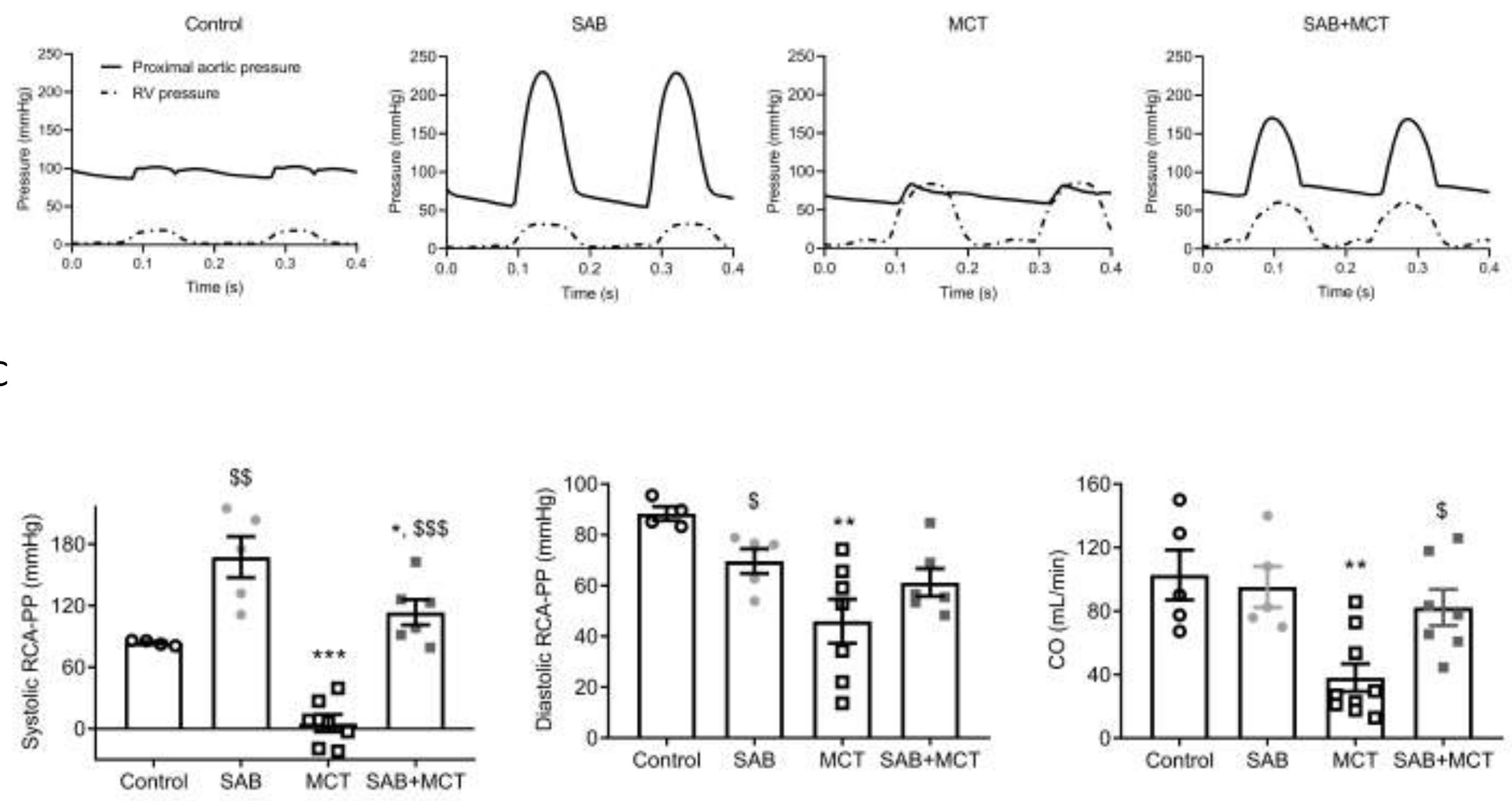

C 
Figure 4. LV and RV end-systolic elastance $\left(E_{e s}\right)$, effective arterial elastance $\left(E_{a}\right)$, and ventricularvascular coupling
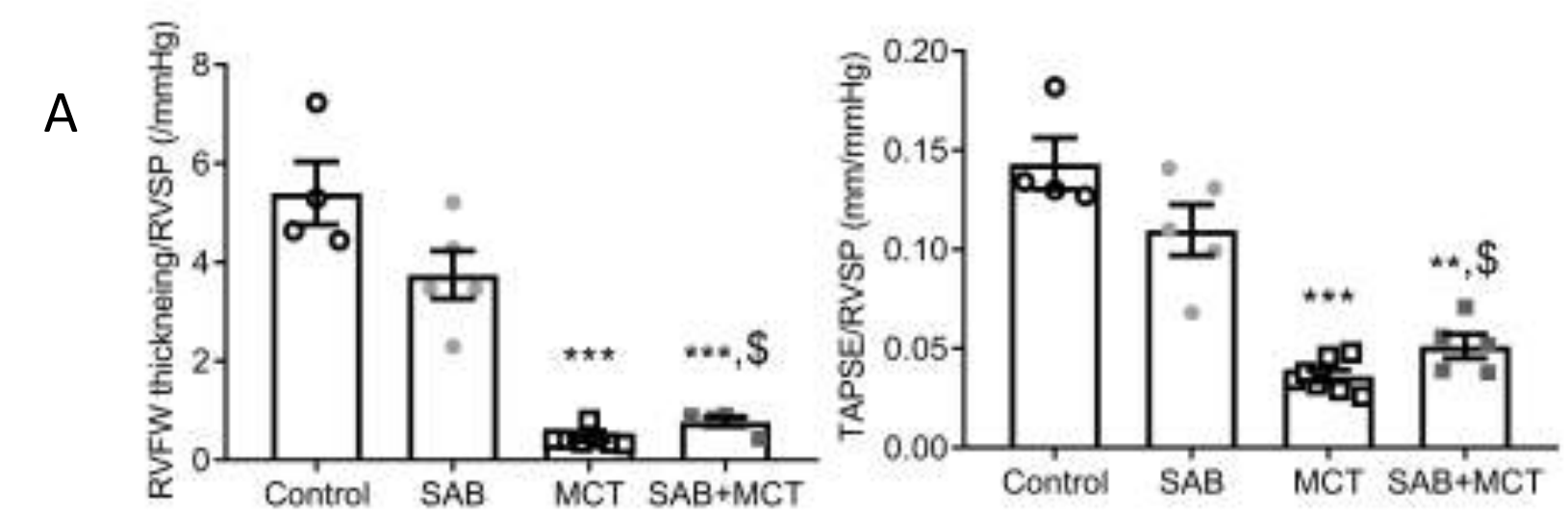

B

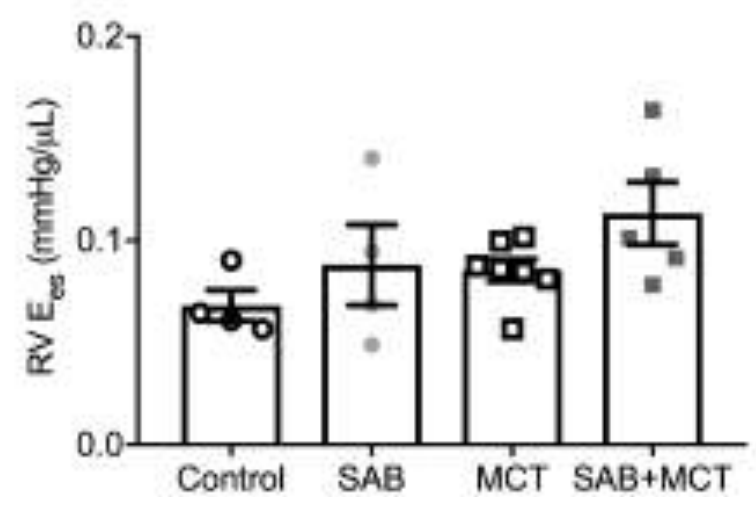

C

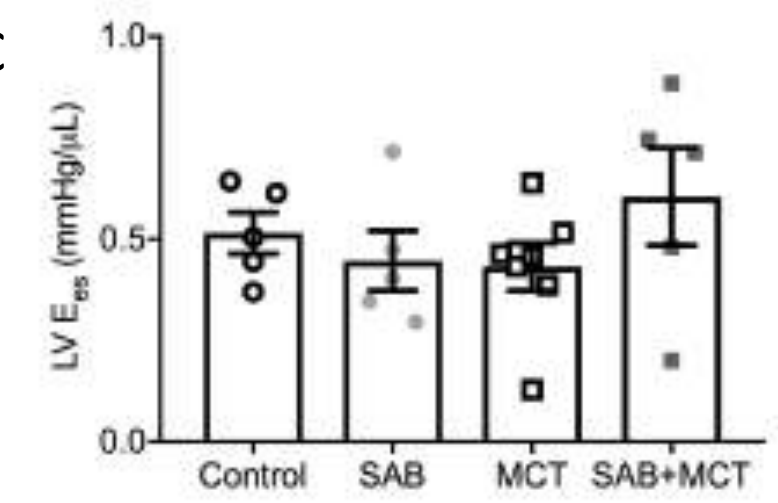

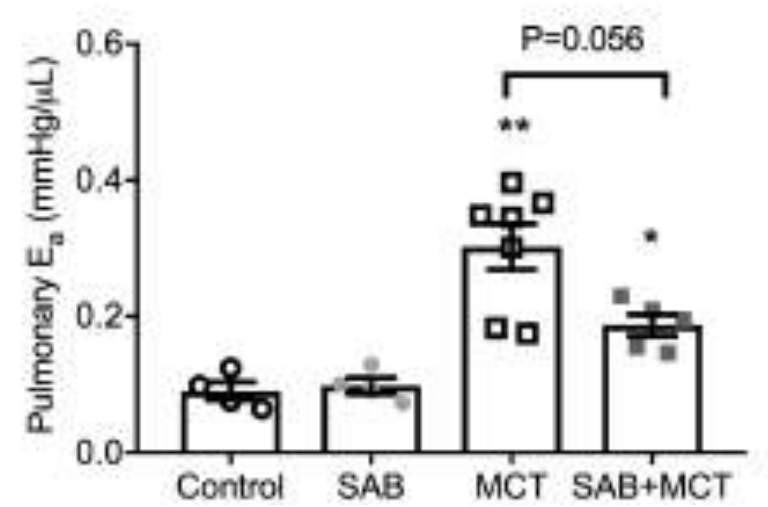
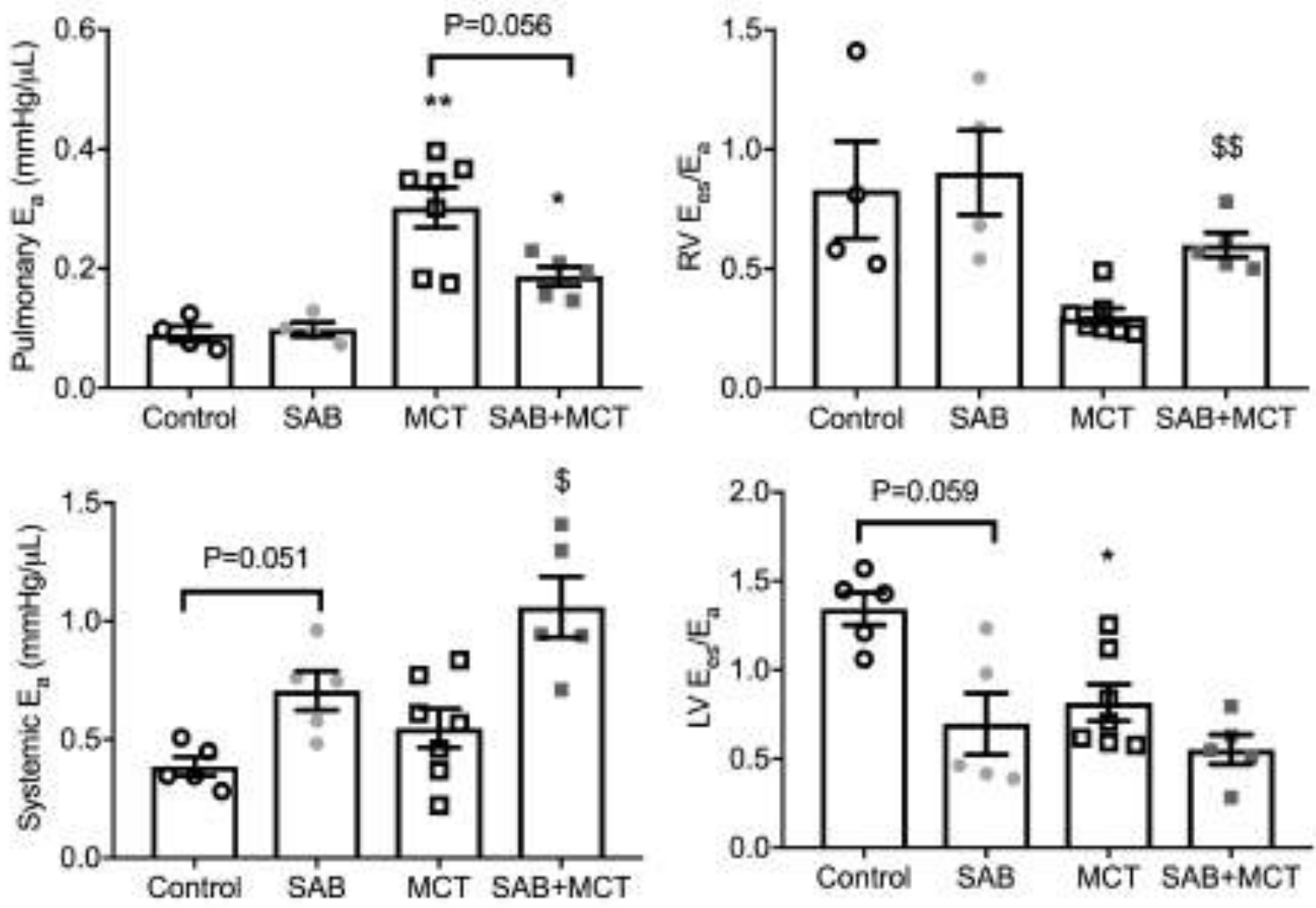
Figure 5. RV contractility indices and CO vs. RCA-PP
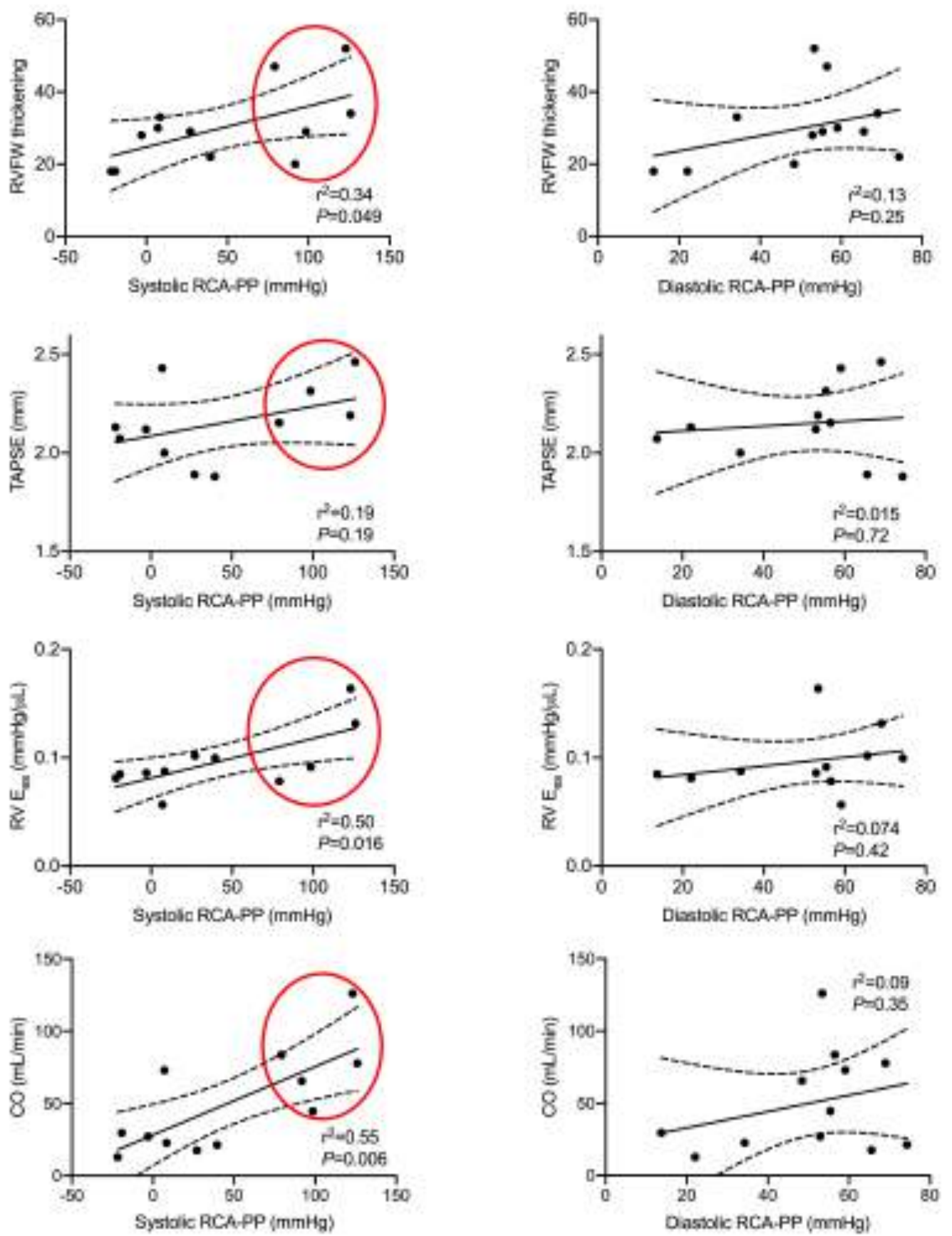
Figure 6. (B) CO vs, radioactivity uptake in RV from MCT rats
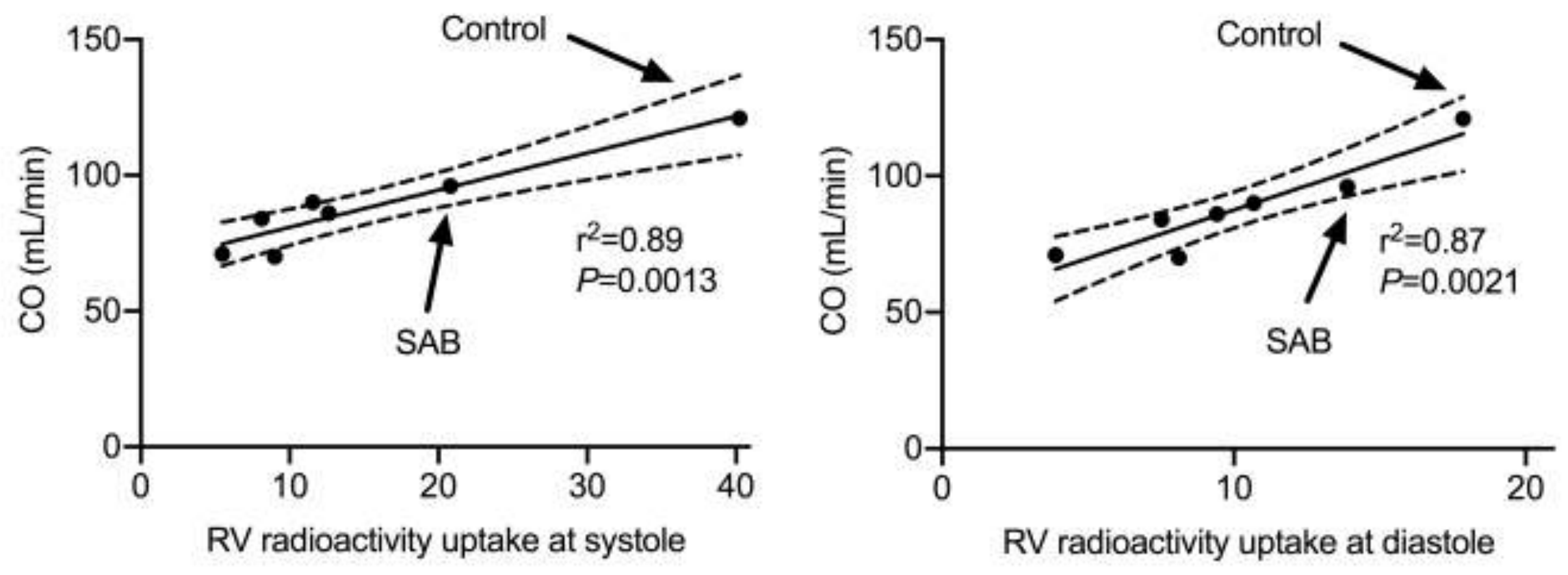
Figure 6. (C) Representative images of microspheres (green) in right ventricular free wall (RVFW)

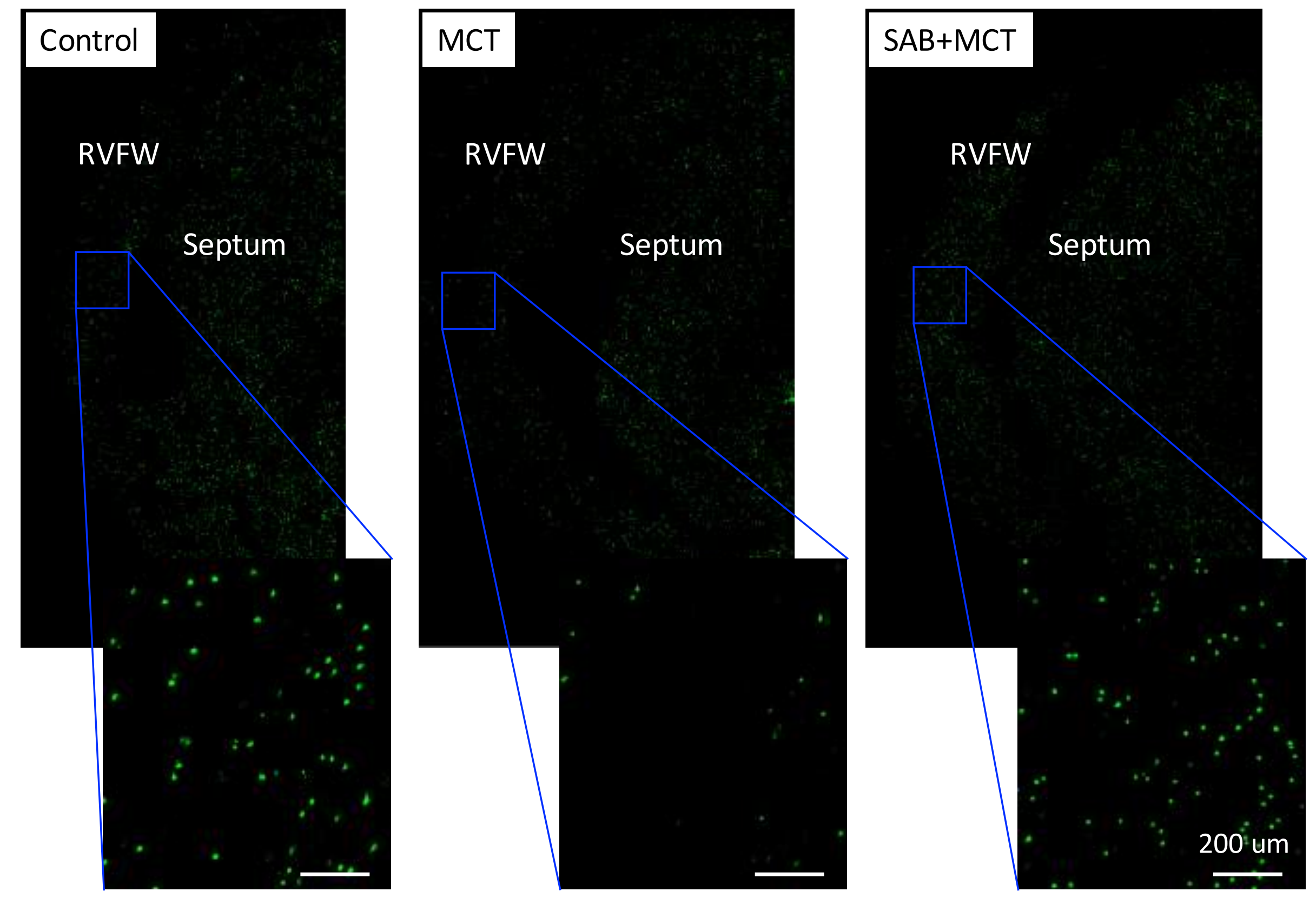


Figure 6. (D) Summary of the number of microspheres in the right ventricular free wall (RVFW)

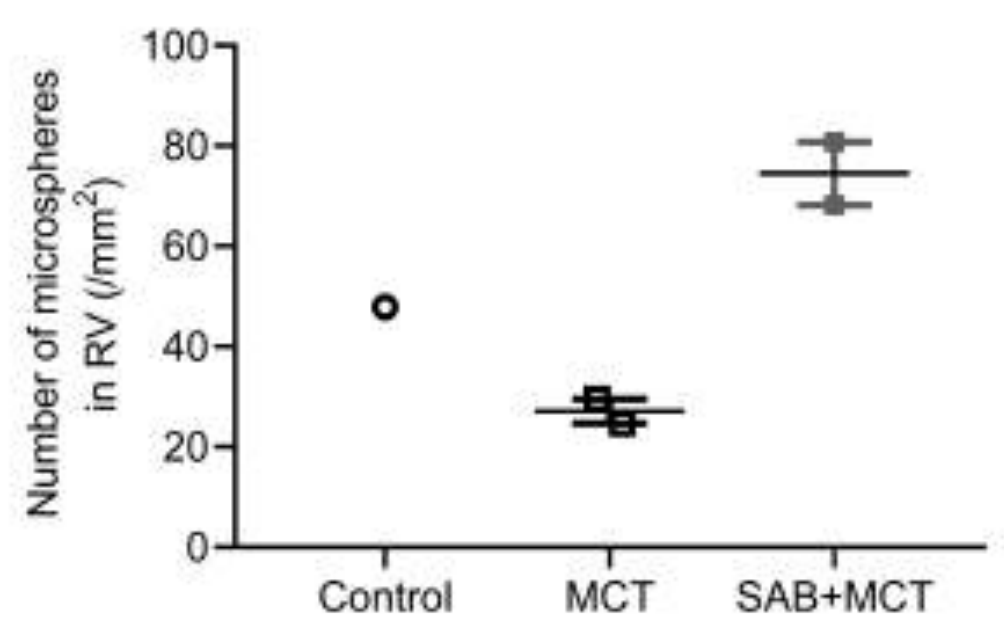



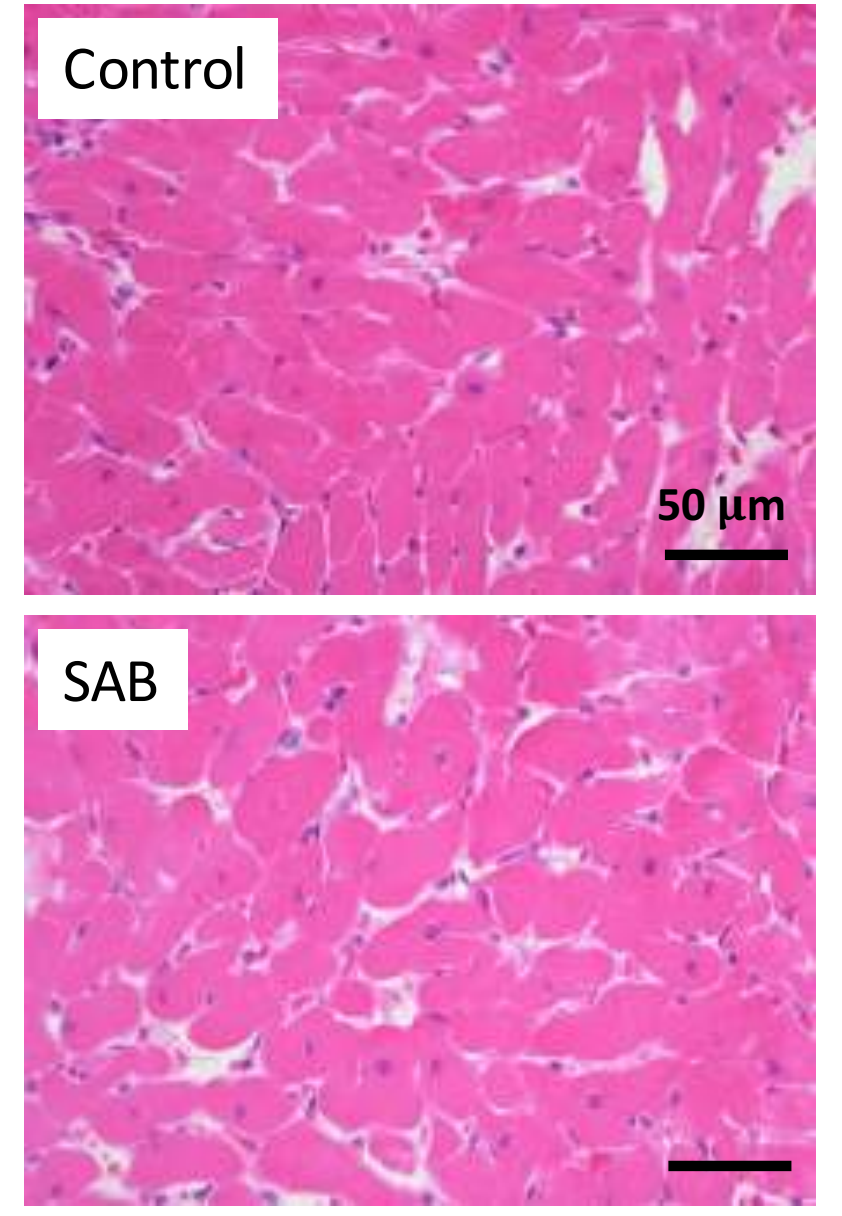
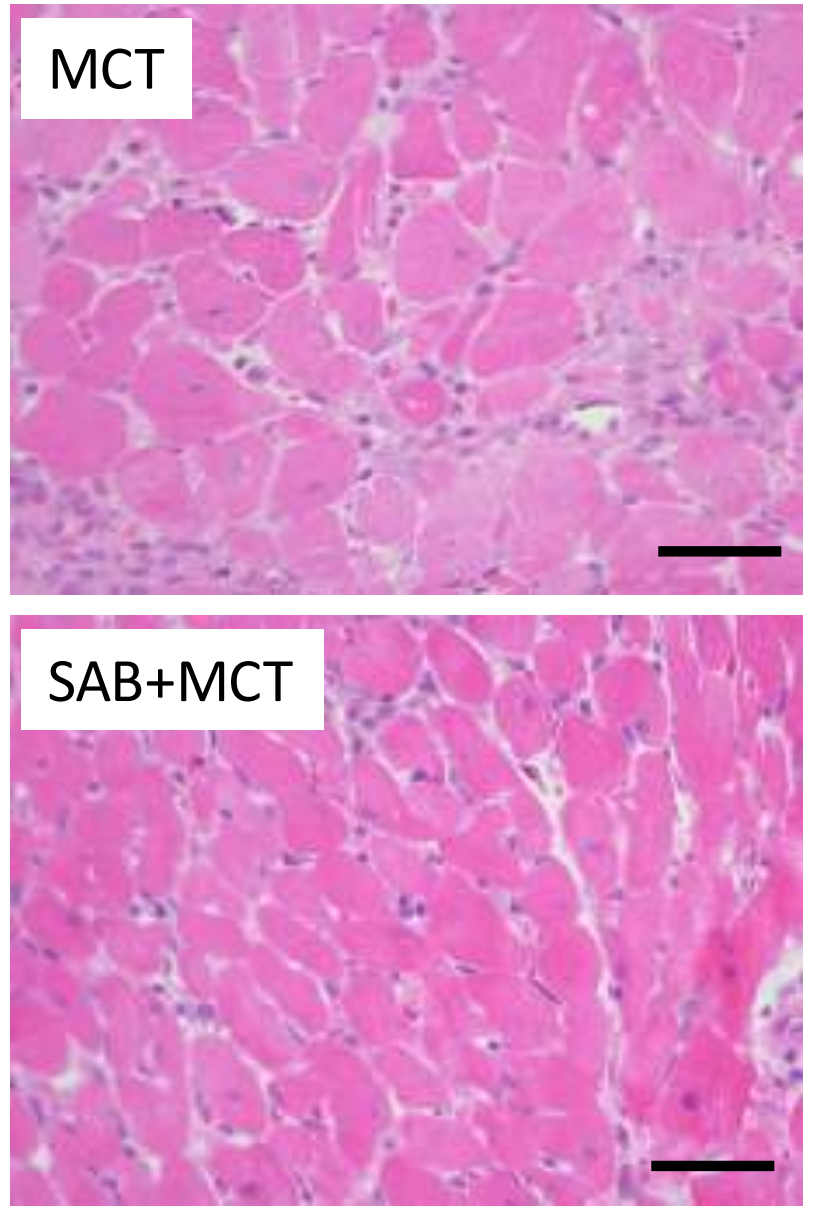

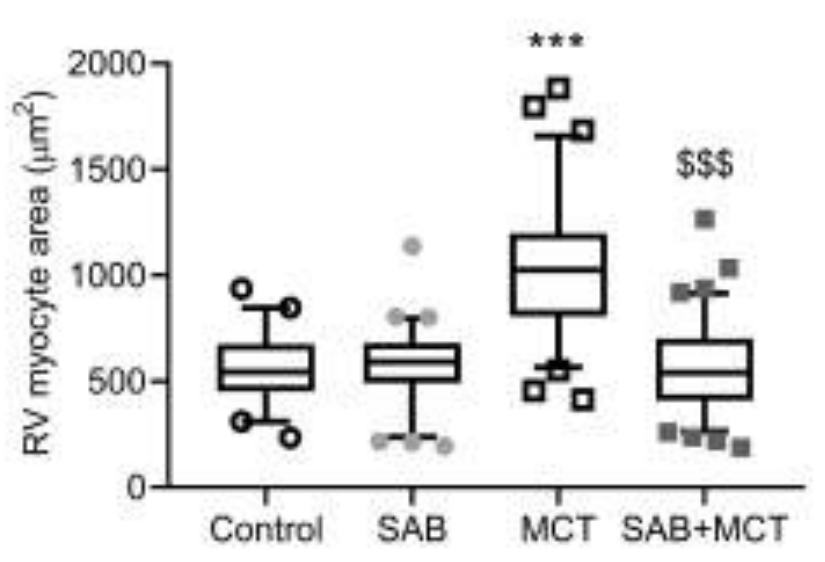


Figure 7. (C) CD31 in RV

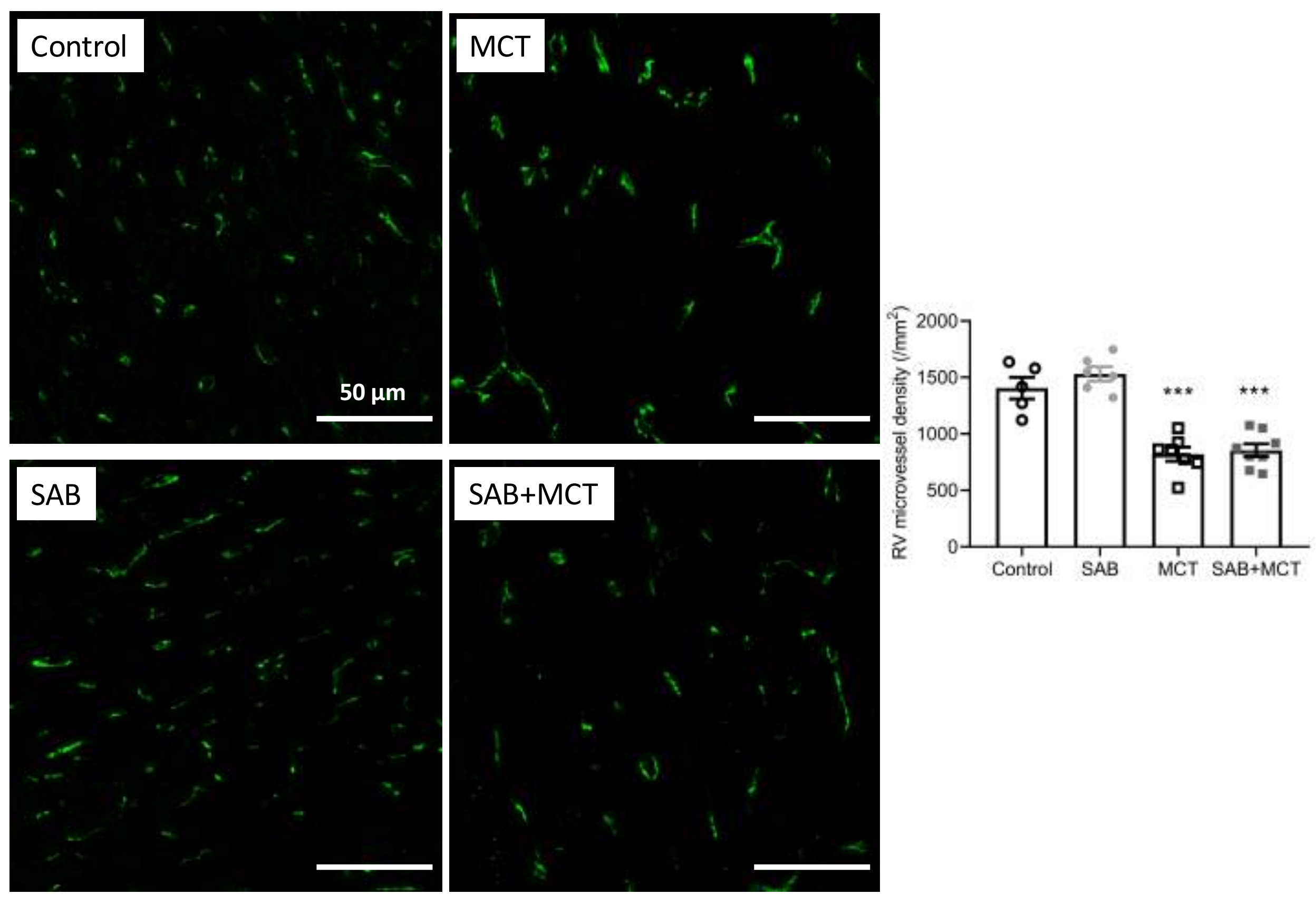


Figure 7. (D) Muscularized pulmonary vessels in the lung

$\begin{array}{llll}\text { Control } & S A B & M C T & S A B+M C T\end{array}$
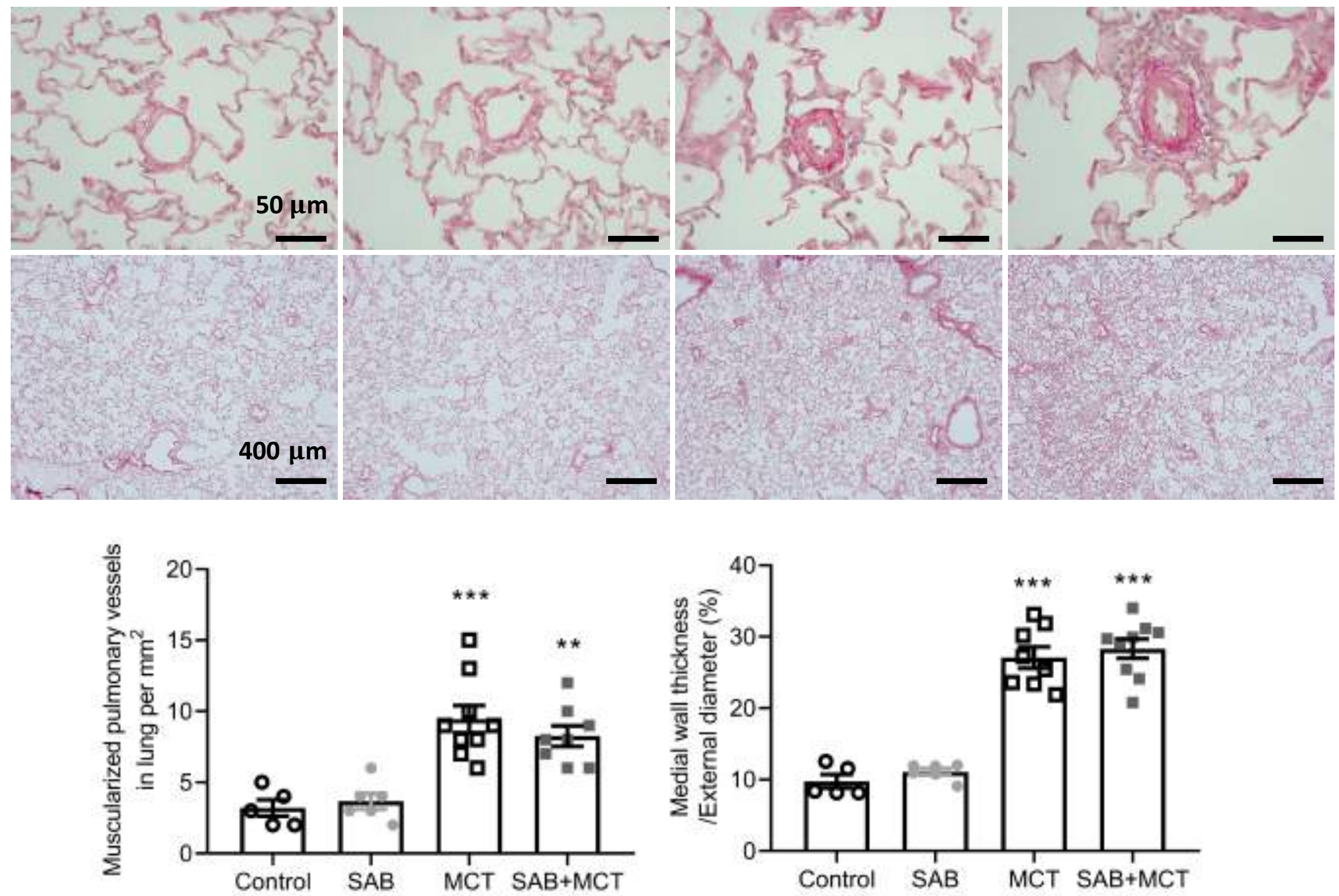
Figure $8 . \mathrm{RV}$ protein measured by western blot
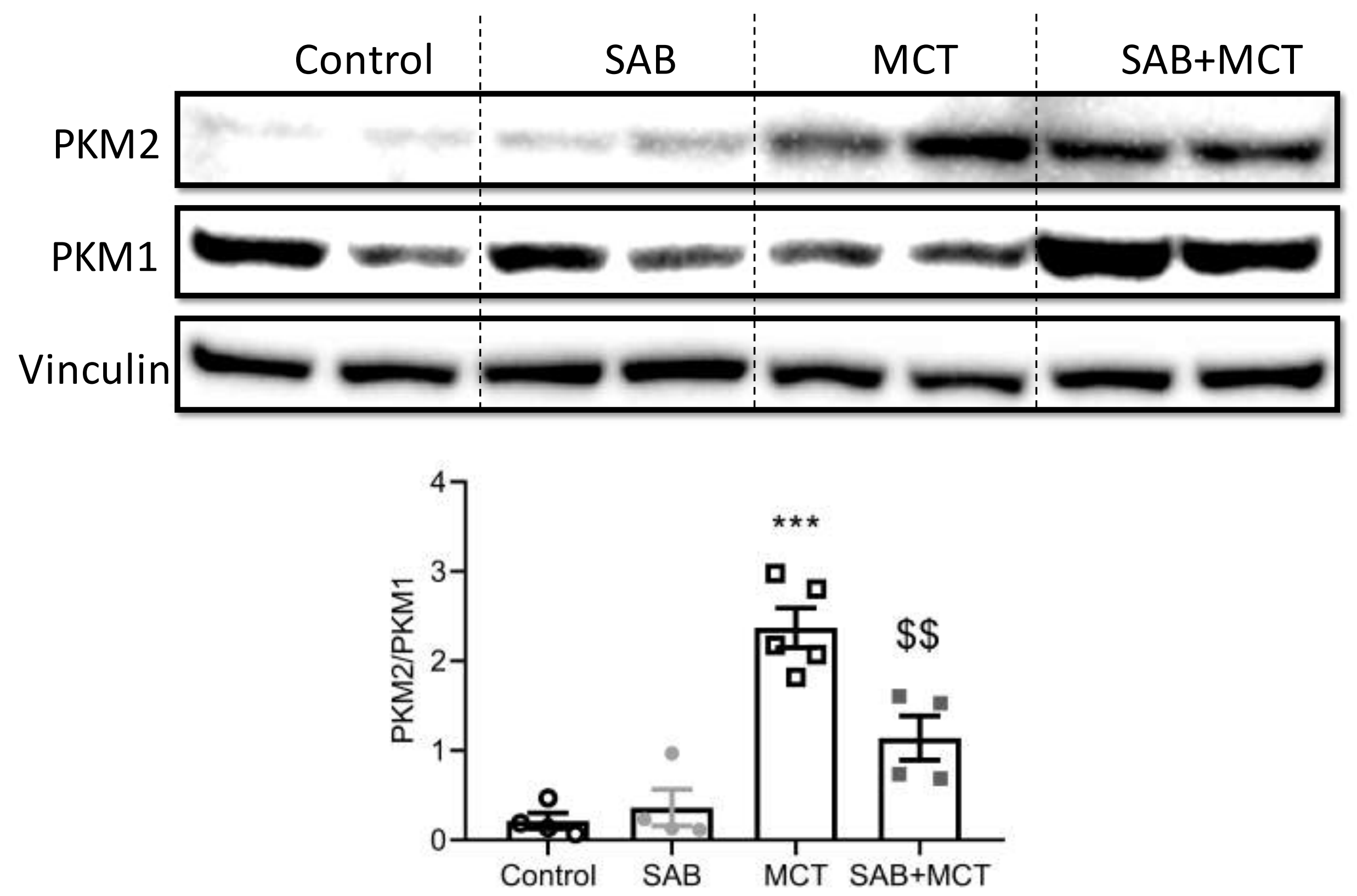